\title{
Effects of Additives upon Percolation Temperature in AOT-Based Microemulsions
}

\author{
A. Cid ${ }^{a}$, O.A. Moldes ${ }^{b}$, J. Morales ${ }^{b}$ and J.C. Mejuto, ${ }^{\text {b. }}$ \\ ${ }^{a}$ REQUIMTE, Departamento de Química, Faculdade de Ciências e Tecnologia, Universidade Nova de \\ Lisboa, Quinta da Torre, 2829-516 Monte de Caparica, Portugal \\ ${ }^{b}$ Department of Physical Chemistry, Faculty of Sciences, University of Vigo, Ourense, Spain
}

\begin{abstract}
In the present review the percolative phenomena has been analyzed. Percolation is related to transport phenomena in microemulsions, in particular the electric charge transport. The influence of different additives upon electric percolation has been commented. The effects of the additives considered upon the microemulsion properties appear to come about through their association with the surfactant interface. The effects of these agents on the ease with which interdroplet channels allowing transfer of droplet contents are formed are not only responsible for their effects on percolation temperature, but also have serious implications for the rates of fast chemical reactions performed in microemulsions.
\end{abstract}

Keywords: Microemulsion, Surfactants, Transport phenomena, Percolation, Aditives, Percolation Temperature.

\section{INTRODUCTION}

Colloid science has been the subject of a revolutionary change, from a simple collection of qualitative observations on the macroscopic behavior of some complex systems to a discipline with a very solid theoretical background. Almost all the experimental techniques and theoretical procedures both physical and chemical- have been gradually applied to the study of colloids. These facts give us a general idea of how complex these systems can be.

One of the most important colloidal systems is represented by those substances that, under certain circumstances, can associate in solution and form aggregates of a high number of units. These systems are characterized by the fact that the contact area between the aggregates that can be formed and the medium that contains them is relatively large. These aggregates may appear in various forms -according to the conditions in which they were formed- such as micelles, vesicles, bilayers, microemulsions, etc. All of them present a high interest in practically all the fields of scientific and technological development [1-6].

In particular, the characteristics that these systems present are used to reproduce in vitro certain biological aspects, such as chemical reactions taking place in the cellular area, matter movements in the organism and organic and inorganic transport phenomena within the human body.

*Address correspondence to this author at the Department of Physical Chemistry, Faculty of Sciences, University of Vigo, Ourense, Spain; Tel: +34 988387000 Ext. 87001; Fax: +34 88 837001; E-mail: xmejuto@uvigo.es
Specifically, the study of micellar systems and synthetic vesicles has experienced a strong rise over the last years. Such was the impetus of these studies and their development that nowadays this area of knowledge has acquired its own name, being known as 'Biomimetic Chemistry' [7-8].

Additionally, it is important to point out that the great chemical interest that these systems present derives from their capacity of solubilizing a great quantity of compounds with well-differentiated hydrophobic and hydrophilic properties [9-11]. Therefore, catalysis in these media constitutes an alternative to the phasetransfer catalysis and other techniques for reactions of water-insoluble organic substrates with water-soluble reagents. We must emphasize the great kinetic interest that these systems present either as scalable reactors that may act as catalysts concentrating the reagents in the colloidal core, or as inhibitors separating the reagents into different domains of these systems [1218].

The aim of this paper is to review the behavior of a type of colloidal system aggregates, microemulsions, and the electrical percolation that occurs in them, a phenomenon related with transport of matter or charge that can be influenced by the presence of additives.

\section{MICROEMULSIONS}

\section{Definition and Types of Microemulsions}

Microemulsions or inverse micelles are colloidal aggregates formed by the self-association of substances called tensioactives [19] (organic compounds with a long hydrocarbon chain and a 
hydrophilic head Scheme 1) in solutions whose major solvent is either organic or apolar. This makes the tensioactive molecules form associations that can present different forms depending on the tensioactive type. The hydrophilic heads of the tensioactive molecules would be at the center of the aggregate, while the hydrophobic carbon chains would be left in direct contact with the organic solvent [20].

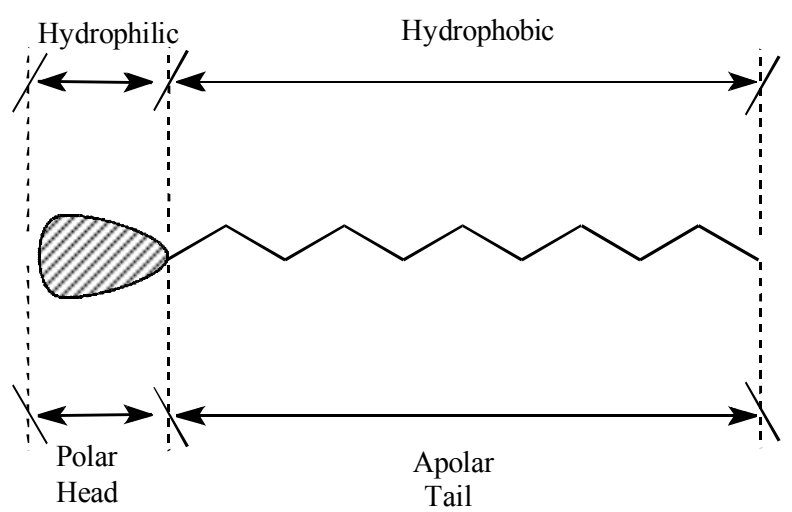

Scheme 1: Organic compounds of long hydrocarbon chain and hydrophilic head.

The most used tensioactive is AOT (sodium bis(2ethylhexyl) sulfosuccinate Scheme 2). This branchedchain tensioactive has the particularity of forming microemulsions with empty volumes in their interior [21]; this empty space can be filled by water alone. The existence of a certain quantity of water in the microemulsion provides a great stability and favors the formation of colloidal aggregates. Therefore, it is advisable the presence of a certain quantity of water in the organic solution in order to obtain stable microemulsions.

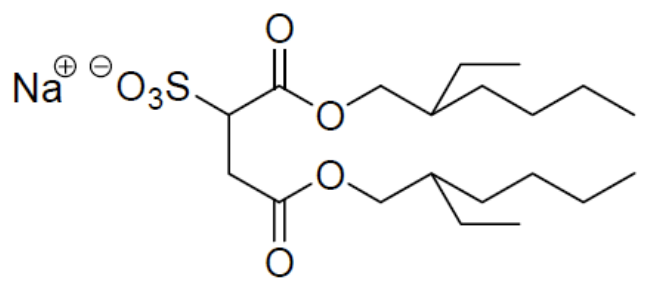

Scheme 2: AOT (sodium bis(2-ethylhexyl)sulfosuccinate).

The most common microemulsions (Scheme 3) are those formed by three phases (organic solvent, water and tensioactive -in some cases it might be necessary the presence of a cotensioactive-) and can adopt various forms and structures depending on the composition of the solution.

Using the Winsor classification system [22 a], we can organize microemulsions in four major groups according to the proportion of their components (see Figure $1[22$ b]):

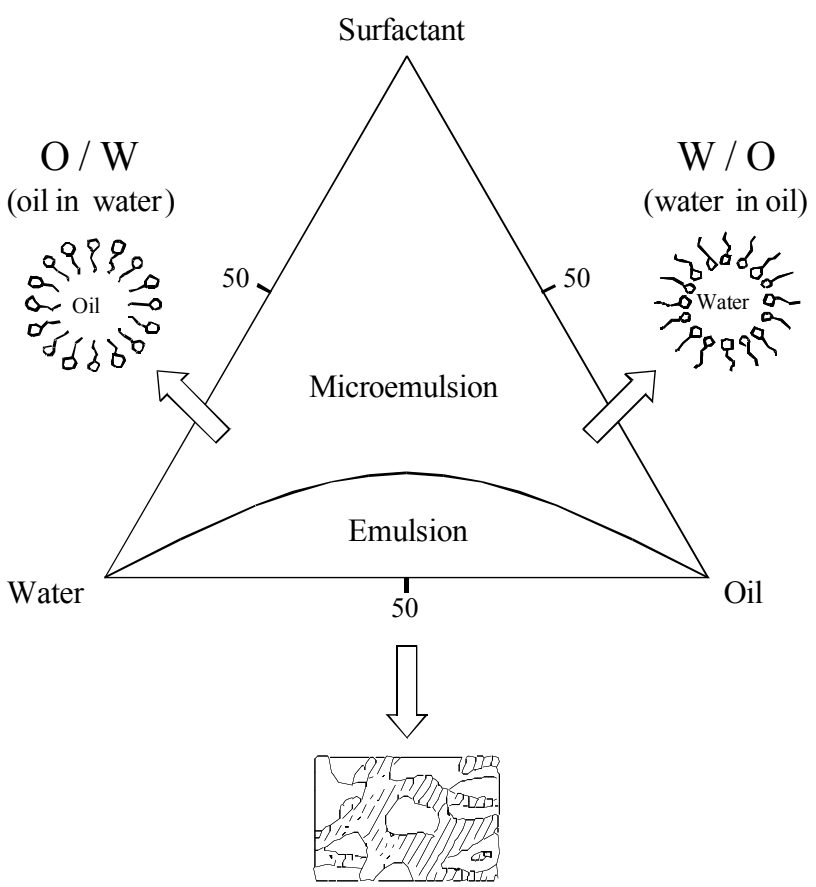

Scheme 3: Phase diagram of ternary system surfactant/ water/oil.

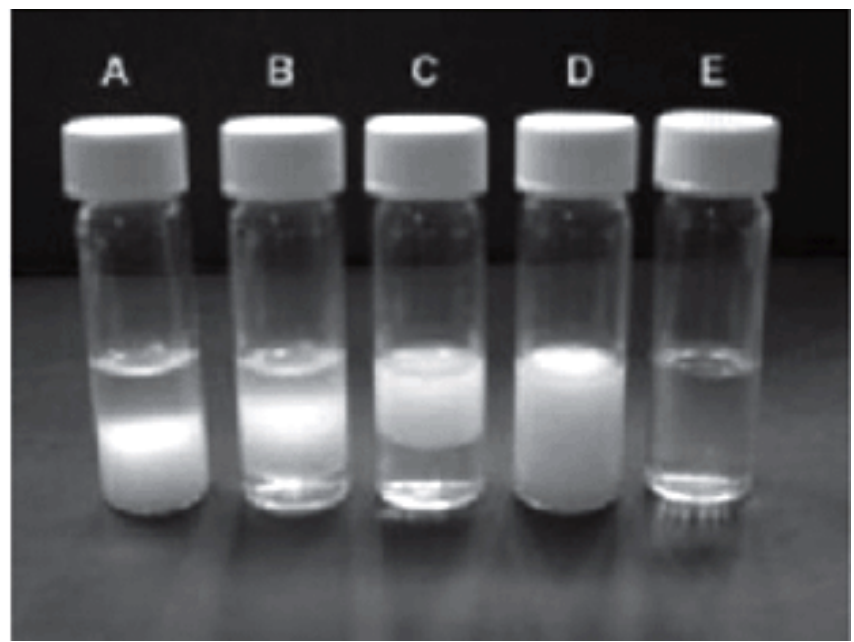

Figure 1: Winsor Classification: A) Winsor I, B) Winsor III, C) Winsor II, D) Homogeneous emulsion, y E) Winsor IV (Microemulsion).

- Winsor I: In this microemulsion the quantity of water is much higher than that of the organic solvent; as a result, the formation of these structures will be limited to microdroplets of organic solvent dispersed in an aqueous medium surrounding the tensioactive molecules. This microemulsion is also known as lower-phase microemulsion. 
- Winsor II: Represents the opposite case: a solution with an organic solvent as its major component. Therefore, there will be water microdroplets in equilibrium with the organic solvent. This microemulsion is also known as upper-phase microemulsion.

- Winsor III: Consists on the so-called middlephase microemulsion that coexists with two phases, an organic and an aqueous one, respectively.

- Winsor IV: Its main characteristic is the fact that the solution components coexist in a sole isotropic phase.

\section{Geometry of Microemulsions}

A series of energetic aspects [23-33] intervene in the formation of microemulsions that will adopt one aspect or another. The result is the same in all cases: finding a geometry in which the sum of all the factors involved in the microemulsion energy is as small as possible.
Among the interactions that take place between the tensioactive molecules and the medium where they are located, there are two main groups: i) hydrophilic interactions between the polar head and water and ii) hydrophobic interactions between the hydrocarbon chain and water [21]. The first tends to increase the area of the polar head and the second to decrease it. The equilibrium between both takes place when the energy of the microemulsion for the ideal area $\left(a_{0}\right)$ is minimum.

Besides the optimum area, two other important concepts in the geometry of microemulsions must be taken into account: volume $(v)$ and critical length of the hydrocarbon chain of the tensioactive $\left(I_{c}\right)$ [34]. These three important factors can be calculated by means of empirical equations. The utility of these three aspects resides in a geometry model created by Ninham et al. [35-36] where it is suggested that the result of the previously mentioned interactions can be interpreted by taking into account only geometric concepts. Ninham proposes a model according to which the geometry of the aggregates depends on the value of an expression called packing ratio (Equation 1, being $v$ the molecular

Table 1: Colloidal Aggregate Structure According to the Surfactant Geometry and $\xi$

\begin{tabular}{|c|c|c|c|}
\hline Tensioactive & $\xi$ & Packing Critical Form & Structure \\
\hline Simple Chain and large headgroups & $<0.3$ & & Spherical micelles \\
\hline Simple chain and small headgroups & $0.3-0.5$ & & Cylindrical micelles \\
\hline Double chain and large headgroups & $0.5-1$ & & $\begin{array}{c}\text { Flexible bilayers } \\
\text { Vesicles }\end{array}$ \\
\hline Double chain and small headgroups & $\sim 1$ & & Flat bilayers \\
\hline Double chain tensioactives and small headgroups & $>1$ & & $\begin{array}{l}\text { Reverse micelles } \\
\text { Microemulsions }\end{array}$ \\
\hline & & Inverted & \\
\hline
\end{tabular}


volume of the tensioactive, $a_{0}$ the area of the polar headgroup of the tensioactive, and $I_{c}$ the critical length of the carbon chain of the tensioactive).

$\xi=\frac{v}{a_{0} l}$

According to this model, the curvature [37-39] is related to the micelle radius $(R)$; thus, the micelle radius must be shorter than the maximum length of the hydrocarbon chain. The empiric calculations lead to an expression that can only be valid within the principles of this theory if the mentioned above packing ratio is not higher than $1 / 3$. Otherwise, it would be theoretically impossible to form spherical micelles. If the value of this ratio exceeded $1 / 3$, tensioactive molecules would adopt different forms. Using this equation, we can find the conditions that relate the curvature parameter of the tensioactive and the type and structure of the resulting aggregate.

This classification can be seen in Table 1 as an schematic representation, along with the possible favorable structures that would be spontaneously formed in the presence of water or apolar organic solvent [40-41].

The curvature of the tensioactive film, $C_{0}$, will be taken positive in the case of micelles, and negative in the case of microemulsions. When it reaches a noticeable value, one of the two phases -the organic or the aqueous- forms a continuous medium that presents

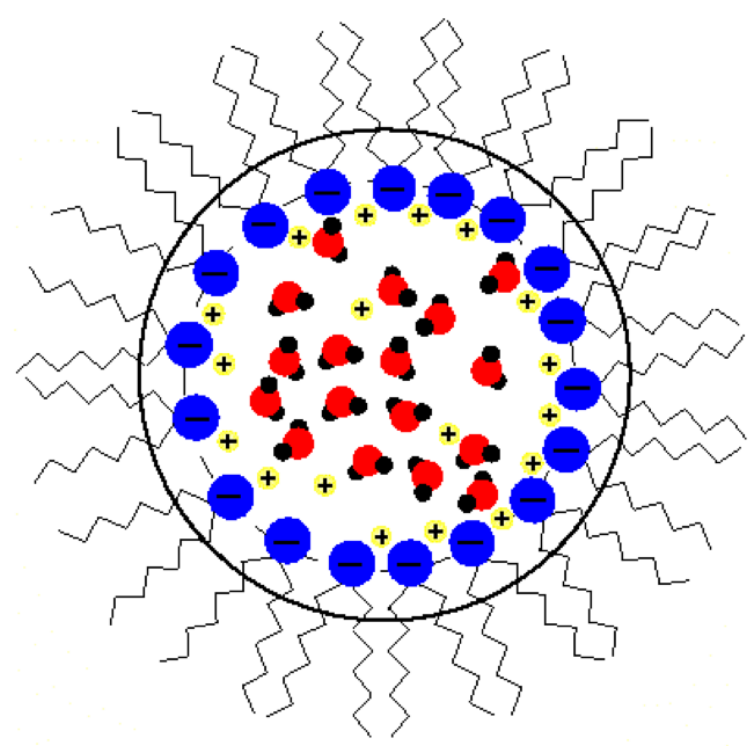

Scheme 4: Charge distribution within microdroplet of microemulsion system according to "microdroplet model" microdroplets of the other component; when dealing with a null value of $C_{0}$, the phases of the continuous medium do not differ from one another [42-43].

As already seen, the length and form of the carbon chain have a direct influence on the packing ratio of the tensioactive. Therefore, a short carbon chain tensioactive and a large polar head, as SDS (sodium dodecyl sulphate) Scheme 5a will adopt a conical structure whose $\xi$ is $<1 / 3$, while AOT Scheme $5 \mathbf{b}$ with a large branched carbon chain and a more reduced polar head will adopt the form of a truncated cone whose $\xi$ is $>1$.

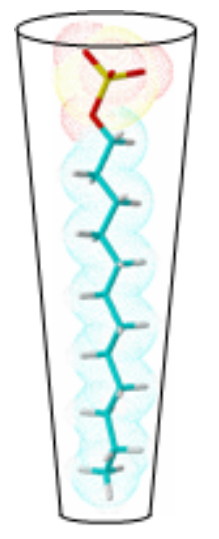

a

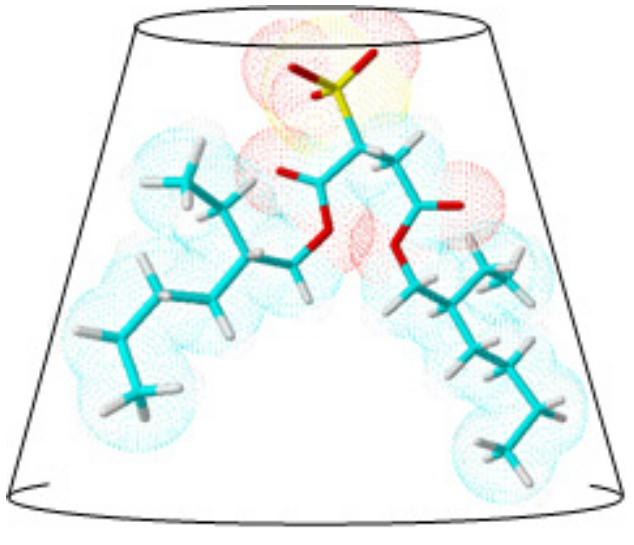

b
Scheme 5: a. SDS geometry $(\xi<1 / 3)$. b. AOT geometry $(\xi>1)$.

The structure and properties of microemulsions can be modified by means of system variations (such as temperature, salinity, presence of unsaturations, etc.) in which the microemulsion is located [11,44-57]. First, the increase of a physical property such as temperature produces noticeable changes in the microemulsion due to the fact that an increase of the carbon chain mobility is generated, originating a decrease of its efficient length and therefore producing an enhancement of the packing ratio. If salts are added, their effect will depend on the salt concentration in such a way that the tensioactive solubility in water will increase at low salt concentrations, while at moderately high salt concentrations its solubility will increase in an apolar organic solvent. Although the behavior is similar, the explanation differs according to the type of used tensioactive. For example, in the case of ionic tensioactives, the increase of the solubility will produce a screening repulsion between the polar heads [58-59] that generates a decrease of the effective area that produces an increase of the curvature parameter-giving rise to microemulsions. However, in the case of non-ionic tensioactives, the 
result can be explained by the fact that the high salinity of the medium stores much of the quantity of water molecules, decreasing the solubility in water of these compounds. Finally, the packing ratio can be modified by varying the number of unsaturations of the carbon chains in such a way that their enhancement (especially if they are cis-type chains) will produce a decrease in the chain length, increasing the curvature parameter that gives rise to the formation of microemulsions of considerable sizes.

\section{Structure of Microemulsions}

Several models have been proposed in order to explain the internal structure of microemulsions; none of which explains their behavior in all situations, as it has been experimentally verified. The most accepted theoretical model is the 'droplet model' [61] which perfectly explains the results obtained experimentally at low concentrations. However, when the compounds concentrations at two phases are similar, other models must be taken into account [61-62]. The droplet model, though not perfect and not valid for all cases, is very useful because it can be easily interpreted. This model considers the dispersed phase to be formed by spherical droplets surrounded by tensioactive molecules; this assumption has been experimentally verified [63-70] when the concentration of the dispersed phase is low.

We assume a microemulsion whose continuous phase is an alkane (organic). If the ratio between water and tensioactive concentrations $\left(W=\left[\mathrm{H}_{2} \mathrm{O}\right] /[\mathrm{AOT}]\right)$ is constant, the composition of the disperse phase also is. If we keep $W$ constant, the size of the droplets will not vary either (though we change the tensioactive composition) since the area of each tensioactive molecule does not vary [71]. As an application of the droplet model and taking into account this reasoning, if we dilute a microemulsion the size of the droplets will remain constant, varying their volume fraction. If we assume that the droplets are spherical and we use the standard volume values of the water molecule [21] and the surface of the polar heads, we obtain a ratio between the droplet radius and the $W$ parameter $\left(r_{w}=1.5 W\right)$.

When the water and surfactant concentrations increase, the droplet model is not useful anymore since it cannot satisfactorily explain the separation of the microemulsion into two isotropic phases, in order to form either a $w / 0$ microemulsion in equilibrium with water (or an $o / w$ in equilibrium with alkane) or two microemulsions $(o / w$ or $w / 0)$. In the first case, a dilution takes place along the phase separation line; the droplets keep the size constant, which will be the largest size possible [72]. In the second case, when two microemulsions are formed in equilibrium, the phase separation occurs via interactions within the dispersed phase. In the case of the droplets, if important attracting interactions take place, the sphere interaction models require phases and therefore, the dispersion separates into two with different sphere concentrations, while the droplet size is the same for the two systems.

The formation of microemulsions often requires a cotensioactive (usually a short-chain alcohol) that is distributed between the droplet interfaces and the continuous phase. At the interface, the tensioactive molecules increase the film density decreasing the interfacial energy [60] so that it becomes more fluid. Considering that the alcohol is also distributed throughout the continuous phase, the phase will mainly contain alkane and alcohol, the water and tensioactive quantities being very reduced [73]. The droplet core will contain pure water, while the rest of the tensioactive and alcohol forms an interfacial film.

If the droplet model is valid, the function whereby the phase separation at a constant size droplet occurs must be linear. It has been experimentally confirmed [42] that for high alkane concentrations straight lines are found, whereas for systems with lower alkane concentrations curves are observed. This fact implies that there is a change in the droplet size in this curved fragment and hence, the droplet model will only be valid for the linear zone. When the water concentration is high, the Talmon and Prager model [61] adjusts better to the experimental results; in this model, the alkane and water play a similar role so that, on changing the quantity of the continuous phase, the area of the interfacial film also varies. Finally, from the measures performed for this type of systems, it can be observed that the droplet size depends on the ratio between the alkane/tensioactive or water/tensioactive, depending on the microemulsion type and increasing the droplet sizes on increasing this relation. It can also be said that the droplet size increases on decreasing the cotensioactive chain [72].

\section{Transport Phenomena in Microemulsions}

Among the physical processes that take place in microemulsions there are two of great interest, which are believed to be able to affect the rate of chemical 
Table 2: Aggregation Numbers of AOT Microemulsions and Radii of the Water Droplets in Different Alkanes and at Different Temperatures. [AOT] $=0.182 \mathrm{M} ; W=26.3$

\begin{tabular}{|c|c|c|c|c|c|c|c|c|c|c|c|c|c|c|}
\hline \multirow[b]{2}{*}{$T^{\circ}{ }^{\circ} \mathrm{C}$} & \multicolumn{2}{|c|}{$n$-Hexane } & \multicolumn{2}{|c|}{$n$-Heptane } & \multicolumn{2}{|c|}{$\begin{array}{c}n \text {-Heptane }+n- \\
\text { Octane }\end{array}$} & \multicolumn{2}{|c|}{ Isooctane } & \multicolumn{2}{|c|}{$n$-Octane } & \multicolumn{2}{|c|}{$n$-Decane } & \multicolumn{2}{|c|}{$n$-Dodecane } \\
\hline & $N$ & $R / A$ & $N$ & $R / A$ & $N$ & $R / A$ & $N$ & $R / A$ & $N$ & $R / A$ & $N$ & $R / A$ & $N$ & $R / A$ \\
\hline 10 & - & - & - & - & - & - & - & - & - & - & 450 & 43.9 & 450 & 43.9 \\
\hline 15 & - & - & - & - & - & - & 426 & 43.1 & 440 & 43.6 & - & - & 510 & 45.8 \\
\hline 18 & - & - & - & - & - & - & - & - & - & - & - & - & 535 & 46.5 \\
\hline 20 & - & - & 409 & 42.6 & 417 & 42.8 & 431 & 43.3 & - & - & 53 & 46.4 & - & - \\
\hline 25 & 402 & 42.3 & 405 & 42.4 & - & - & 467 & 44.5 & 475 & 44.7 & 590 & 48.1 & - & - \\
\hline 30 & - & - & - & - & - & - & - & - & - & - & 850 & 54.3 & - & - \\
\hline 35 & - & - & 420 & 42.9 & 486 & 45.1 & 521 & 46.5 & 556 & 47.1 & 740 & 51.8 & - & - \\
\hline 40 & 426 & 43.1 & - & - & - & - & - & - & - & - & 580 & 54.3 & - & - \\
\hline 45 & - & - & 465 & 44.4 & 568 & 47.5 & 628 & 49.1 & 670 & 50.2 & 955 & 56.4 & - & - \\
\hline 47.5 & - & - & - & - & - & - & - & - & - & - & 1050 & 58.3 & - & - \\
\hline 50 & 476 & 44.8 & 492 & 45.3 & - & - & - & - & - & - & & - & - & - \\
\hline 55 & - & - & 526 & 46.3 & - & - & - & - & - & - & - & - & - & - \\
\hline 60 & 531 & 46.4 & 554 & 47.1 & - & - & 784 & 52.9 & 930 & 56.0 & - & - & - & - \\
\hline
\end{tabular}

processes close to the diffusion control. These processes are the matter transport and the electric charge transport.

\section{Matter Transport}

The first studies carried out on the subject date back in 1976; these studies confirmed the existence of a mechanism through which a substance exchange takes place between the droplets that form the microemulsions. According to the study, this exchange takes place due to a collision between similar droplets. The studies on matter exchange were carried out by using quenching techniques of fluorescence resolved in time or by kinetic stopped-flow measurements. According to the first technique, Atik and Thomas [74] studied the reagent exchange between droplets by using the quenching of the fluorescence of pyrene sulphates by Fermi's salts or $\mathrm{Cu}^{2+}$ ions. They obtained similar results when using both Fermi's salt and $\mathrm{Cu}^{2+}$ salt. In order to explain the exchange process, they considered that a collision takes place between droplets, the ion transport occurring when mixing their content. In Table 2 it can be seen the effect of different additives on the bimolecular rate constant for the exchange process.

It has been confirmed that the most effective additive in favoring the matter exchange between droplets is the benzylic alcohol that increases the exchange rate approximately 30 times. It is supposed that this alcohol is located in the water-alkane interface causing a disorganization of the tensioactive headgroups. This has repercussions on the rate of matter exchange between droplets once they come into contact with each other.

In the case of other molecules that exert a similar effect on the interface, it has been found an analogous behavior, although the rate enhancement is lower. However, tetramethylammonium and $\mathrm{Na}_{2} \mathrm{SO}_{4}$ decrease the exchange rate because they increase the cation bond to the tensioactive headgroups causing a higher rigidity of the surfactant film.

Another more comprehensive study has been carried out by Robinson et al. [75] by means of the stopped-flow spectrophotometric technique in which they used ionic substances to ensure they would be dissolved in water droplets. In order to explain the exchange of these ionic substances between water droplets two possible mechanisms were proposed (Scheme 6a and 6b).

In the scheme (Scheme $\mathbf{6 a}$ and $\mathbf{6 b}$ ) $K_{\text {chem }}$ is the second order rate constant of the reagent exchange between droplets, and $K_{\text {dis }}$ is the rate constant of the dissociation of the dimer droplet.

In order to guarantee that the process of reagent exchange between the droplets is rate-limiting, these authors chose reactions in which the chemical process 
a

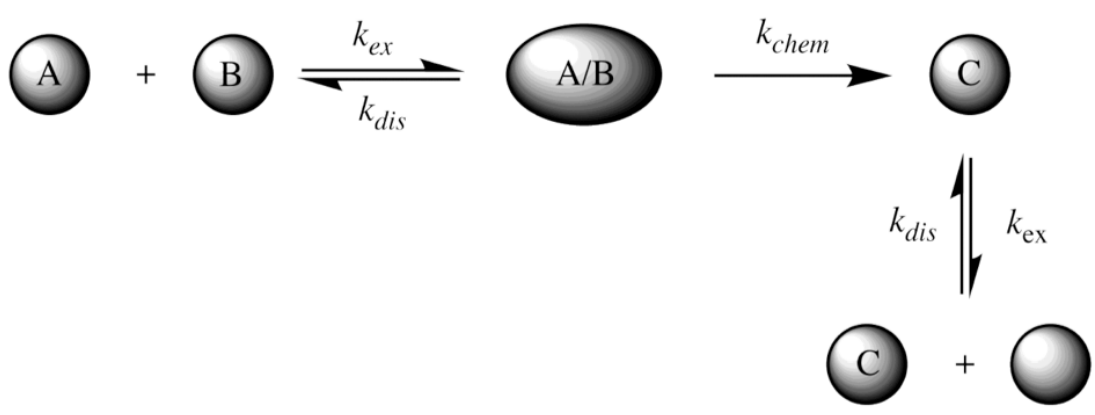

b

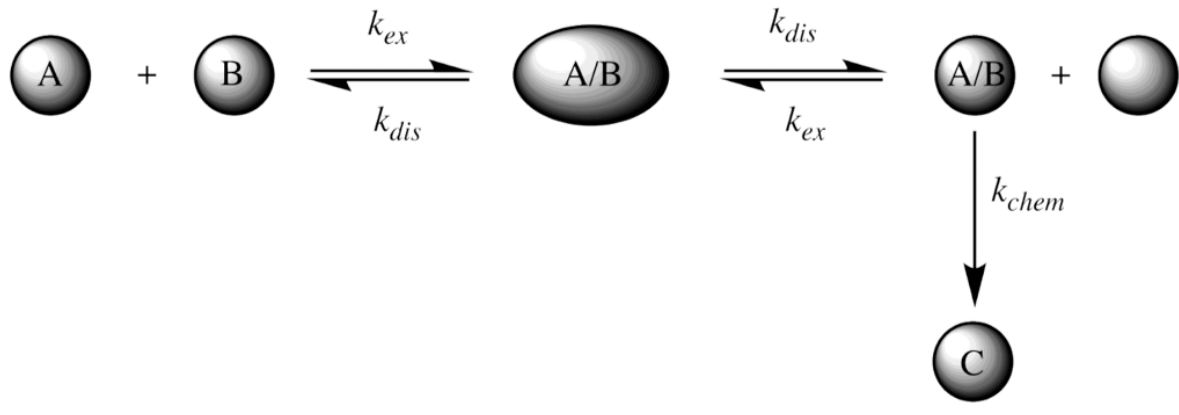

Scheme 6: a,b.

was close to the diffusion control, operating at low droplet concentrations. Under these considerations, it has been supposed that the scheme of the operating reaction must be the $\mathbf{6 b}$.

It has been observed that the values of the exchange constant, $K_{e}$, are independent of the nature of the reaction, the reagent, AOT concentrations, temperature and $W$ constants. These results are concordant with the fact that $K_{\text {chem }}$ is not rate-limiting.

The exchange between the solubilized products could be explained via two mechanisms. The first one involves a joint diffusion of the droplets in order to create an encounter pair, in such a way that the dissolved particles would spread across the tensioactive film in the junction point of the droplets, while these are in contact with each other. Finally, the droplets would separate without having joined together. Due to the fact that the exchange constant is independent of the nature of the exchanging species, this mechanism cannot be valid since, on dealing with a process of permeability through the membrane, the transfer rate would greatly depend on the nature of the substrates.

Another mechanistic possibility would involve the fusion of two droplets, in such a way that the unstable dimmer species lived sufficient enough to allow a random distribution of the droplet content. An intermediate explanation between the previous mechanism and this one could be proposed where the water channels among the droplets could be formed during the life span of the encounter pair. However, these channels should be sufficiently large so that the charged interface could not exert any noticeable effect on the ion migration, reaching the extreme case that postulates the union between droplets. If all the encounters between droplets caused a matter exchange, $K_{\text {exc }}$ would have to be equal to the collision rate. However, the experimental results show that this occurs only in $10^{-3}$ or $10^{-4}$ of the collisions, which suggests the existence of an energetic barrier for the exchange process. The exchange constant will be considered a result of two stages: the association of two droplets in order to generate an encounter pair (prior equilibrium, $K_{\text {enc }}$ ), and the fusion -representing the slow stage- so that $K_{\text {exc }}=K_{\text {enc }} K_{\text {fus }}$, as it can be seen in Scheme 6b.

The fusion of two droplets in contact with each other to create a dimmer involves some distribution in the alkane-water interfacial area. The contraction of the area could generate either the tensioactive desorption in the continuous phase (existing evidences of the 

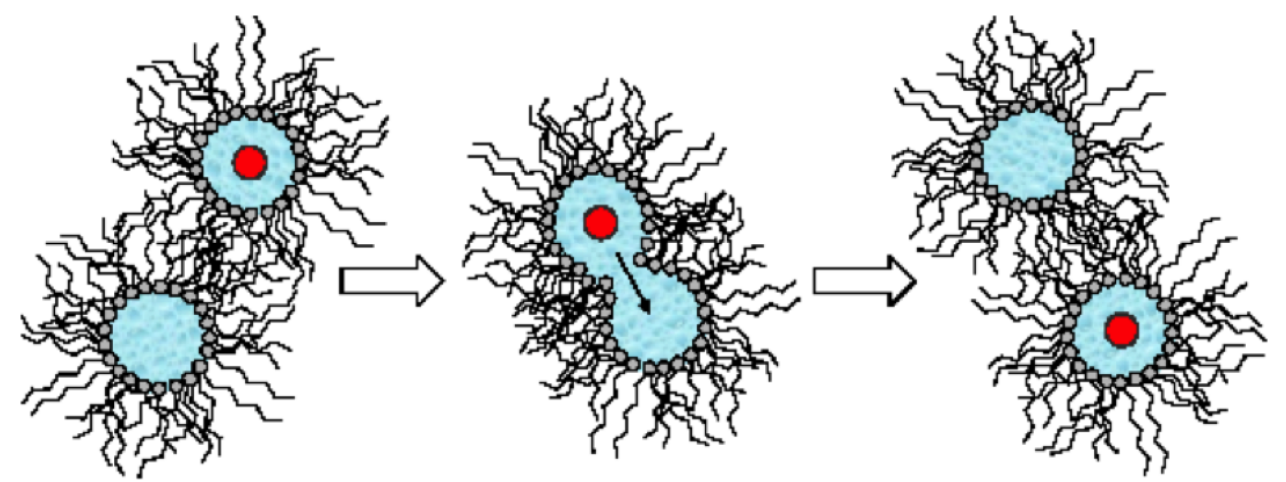

Scheme 7: Proposed mechanism for channels formation between droplets, that eases matter and electronic charge exchange.

presence of small AOT aggregates in the alkane) or the compression of the tensioactive film with the corresponding decrease of the interfacial area occupied by the AOT.

It would be normal to expect that some correlation might exist between the matter exchange and the thermodynamic stability. In fact, the results show that the exchange constant increases with the number of carbon atoms of the alkane, increasing the exchange rate with temperature, which agrees with the fact that the droplets are more stable in the low-temperature zone, where the curvature of the tensioactive film is equal to the natural curvature.

In Scheme 7 possible transition state for the matter exchange can be observed. As shown, there is a highly positive local curvature, i.e., a region where the headgroups have increased their separation at expenses of the separation decrease between the surfactant hydrocarbon tails. On increasing temperature, the enhancement of the positive natural curvature is favored and therefore the exchange is favored. Thus, the effect of temperature on the exchange constant may be rationalized by considering a situation in which it decreases the energetic barrier for the fusion of the droplets, instead of a situation where there is a higher quantity of species with enough thermal energy to exceed the energetic barrier of the reaction. By using the curvature factor, it is possible to carry out a simple interpretation of the effect of additives on $K_{e}$. Thus, the cholesterol and toluene decrease the exchange rate, since they favor the enhancement of the negative curvature at the interface. For the same reasons, when $\mathrm{D}_{2} \mathrm{O}$ is replaced by $\mathrm{H}_{2} \mathrm{O}$, it can be also observed a decrease of $K_{e}$.

Subsequently, Lang et al. [76] studied the structure and dynamics of AOT microemulsions by means of fluorescence resolved in time, obtaining values for the aggregation numbers $(N)$ and the rate constant of the matter exchange between the droplets, $K_{e}$. They studied the influence of temperature, number of carbon atoms of the alkane, AOT concentration, $W$ and addition of electrolytes. They observed that both $N$ and $K_{e}$ increase with temperature and length of the hydrocarbon chain of the alkane. The increase of the aggregation number on increasing the length of the oil chain is interpreted as a consequence of the penetration of the oil molecules in the AOT film. On increasing the alkane length, it becomes more difficult to penetrate in the AOT film, which leads to an increase of the aggregation number. This is equivalent to consider that the curvature radius of the microemulsion

Table 3: Micellar Aggregation Numbers and Water Droplet Radii for Different $W$ Values. [AOT] $=0.182 \mathrm{M} ; T=25^{\circ} \mathrm{C}$

\begin{tabular}{|c|c|c|c|c|c|c|c|c|}
\hline \multicolumn{6}{|c|}{$n$-Decane } & \multicolumn{3}{|c|}{$n$-Heptane } \\
\hline$w$ & $N$ & $R / A$ & $w$ & $N$ & $R / A$ & $w$ & $N$ & $R / A$ \\
\hline 8 & 95 & 17.6 & 26.2 & 555 & 47.0 & 11.4 & 108 & 20.7 \\
\hline 13 & 245 & 28.4 & 26.3 & 590 & 48.1 & 15 & 180 & 26.8 \\
\hline 14.6 & 215 & 28.2 & 26.3 & 670 & 50.2 & 18 & 255 & 32.0 \\
\hline 14.6 & 277 & 30.7 & 26.7 & 592 & 48.4 & 19.5 & 268 & 33.5 \\
\hline 18 & 405 & 37.4 & 29 & 675 & 51.9 & 21.2 & 314 & 36.3 \\
\hline 19 & 425 & 38.7 & 29.8 & 830 & 56.2 & 23.6 & 350 & 39.0 \\
\hline 21.2 & 424 & 40.1 & - & - & - & 26.3 & 405 & 42.4 \\
\hline 23 & 575 & 45.6 & - & - & - & 30. & 520 & 48.3 \\
\hline
\end{tabular}


decreases. In a similar way, when temperature increases, the alkane molecules cannot efficiently pack together at the interface due to the thermal energy and thus, the aggregation number increases. These results can be seen in Tables 2 and 3 .

It is observed that $K_{e x c}$ increases as temperature and number of carbon atoms of the alkane are increased. This is supposedly due to the strength of the attractive intermicellar interactions. The extension of these interactions depends on the relative force of the interactions between the tails of the AOT micelles and between those and the alkane molecules. It is known that the bigger the alkane molecules are, the more difficult is for them to orient parallelly to the AOT chains in order to maximize this interaction. So that, on increasing the size of the alkane, the 'tail-tail' interactions become more important than the 'tailalkane' ones. Thus, on increasing the attractive forces, it will be easier that collisions exist between the micelles and $K_{\text {exc }}$ would increase. When temperature increases, the increase of the thermal energy makes difficult the orientation of the alkane molecules to maximize the 'tail-alkane' interaction, and so they increase the intermicellar forces with the resulting increase of $K_{\text {exc }}$.

When the effect of increasing $W$ is studied by means of keeping AOT concentration constant, an increase of the aggregation it is observed (Table 3 ) as it would be expected from the mentioned increase of the micellar size with $W$.

When salts are added, these authors find a decrease in both $N$ and $K_{e}$. Despite the high degree of dissociation of the tensioactive -which involves a high concentration of $\mathrm{Na}^{+}$in the water droplet- the addition of a small quantity of salt has an important effect. They suppose that the electrolyte addition causes that the micelle become more spherical, so that $N$ decreases and the $\mathrm{v} / \mathrm{s}$ ratio increases when the micelle becomes more spherical, which contrasts with Cabos and Delord work [77] in AOT/heptene/water in the presence of $\mathrm{NaCl}$. They interpret these neutron dispersion results as evidence that salt produces an increase in the size of the aqueous core of the microemulsion.

\section{Electric Charge Transport}

Another phenomenon found in microemulsions is the sudden increase in conductivity on slightly variation of their composition or other physical property (normally temperature). This phenomenon is known as electric percolation.
The first theories on this phenomenon recovered the effective medium theory (EMT), initially developed to explain the properties of metallic dispersions in adequate media. This theory defends that the system is formed by conductive spheres of conductivity $\sigma_{d}$ and radius $r$, and by the rest of spheres with conductivity $\sigma_{\mathrm{m}}$. The effective medium is defined as a medium of conductivity $\sigma$. The theory considers a typical element of the disordered system, which is submerged in an effective medium, resolving the local field right around the above-mentioned element, imposing the condition that the fluctuations of that field around that mean value should be cancelled out. If the effective medium condition is imposed, i.e., the net polarization will be negligible, the following expression for conductivity is obtained:

$\Phi \frac{\sigma_{d}-\sigma}{\sigma_{d}+2 \sigma}+\frac{\sigma_{m}-\sigma}{\sigma_{m}+2 \sigma}=0$

where $\Phi$ is the volume fraction occupied by the conductive spheres. If $\sigma_{m} \approx 0$, as occurs in microemulsions, where the continuous medium is the alkane $(\sigma \approx 0)$, the following expression for conductivity is obtained:

$\sigma=\frac{3}{2} \sigma_{d}\left(\Phi-\frac{1}{3}\right)$

According to this model, only when the volume fraction of the disperse phase is $\Phi=1 / 3$, conductivity begins to increase in such a way that the percolation threshold is situated in the $33 \%$ of the volume occupied by the disperse phase.

The percolation threshold predicted by the EMT does not correspond with the experimental observations, since percolation for volume fractions of disperse phase $\leq 1 / 3$ can be often observed. The theory has been slightly modified. Kirkpatrick [78-79] for instance, considers that the system is formed by a network so that the surrounding nodes are connected via resistors distributed by means of a statistical method. These theoretical studies confirm that the percolation limit of $\Phi_{p}=0.25$ will be eventually reached for a series of resistors randomly distributed on a simple cubic network.

This problem was solved by Webman and Jortner [80] in the case of a continuous medium. The former showed that on increasing the correlation between metallic bonds, the minimum percolation shifts to 0.15 , while the latter studied the possible percolation in a 
cubic network with correlation positions extended to the nearest three, obtaining a value of 0.17 . These results seemed to solve the problem of the EMT.

The possibility of these dipole-dipole interactions will depend on the separation between the conductive particles: if the separation is big enough, it will not be necessary to resort to these interactions, but if on the contrary this does not take place, these interactions will have to be taken into account (this will normally happen on increasing the concentration of the conductive substances). When these substances interact with each other, they form clusters or dispersion chains. This approach carried out by Granqvist and Hunderi [81] is one of the principles of the effective-medium theory with dipole-dipole interaction, EMTDD. The formed aggregates may be considered individual nonspherical entities so that the percolation zone will shift to concentrations lower than those of the conductive material. With a medium of null conductivity, this theory predicts values of 0.271 for structures in chain and 0.156 for clusters.

Other theories that have been developed bring in modifications on the EMT [82], and consider that these dispersions are formed by clusters. The following expression for conductivity is obtained:

$\sigma=1.05 \sigma_{d}(\Phi-0.157)$

Therefore the volume fraction of the disperse phase in the percolation zone would be given by $\Phi_{\mathrm{p}}=0.157$.

Comparing the two fundamental theories, it is observed that when the medium conductivity is null and the volume fraction of the conductor is 0.7 , the EMT and EMTDD become indistinguishable. Both predict a linear ratio between relative conductivity and volume fraction of conductive substances close to the percolation zone; however, no one satisfies totally the observed behavior.

In an extensive study about the behavior of 32 microemulsions regarding the percolation phenomenon, Moulik et al. [83] compared the main theories. In this study, it is observed that when the fraction volume in the disperse phase is small, there is a maximum of conductivity at low percolation temperatures. This effect of volume variation of the disperse phase on percolation temperature is tried to be explained by means of two antagonist effects. It is believed that on increasing the water content an enhancement in the hydration of the ionic pair between the tensioactive and the counterion is produced along with a conductivity increase. Also, an increase of the droplet size produces a decrease of the mobility and concentration of the ionic pair (this effect is important for high water contents).

An alternative theory to explain these experimental facts is the charge fluctuation theory proposed by Eicke et al. [84]. This model is based on the assumption of spontaneous charge fluctuations caused by the anion variation of the tensioactive and counterions in each water droplet. Due to these fluctuations, the droplets will be able to transport charge (both positive and negative), which explains why conductivity is proportional to the volume fraction of the droplets for a wide range of compositions. The model also allows expressing these results through an equation that depends on the droplet radius, reproducing the results experimentally obtained in diluted microemulsions (volume fractions in the range of $0.03-0.05$ and 0.15 $0.3)$.

$\sigma=\frac{\varepsilon_{0} \varepsilon k_{B} T}{2 \pi \eta} \frac{\varphi}{r^{3}}$

where $\kappa_{B}$ is the Boltzmann's constant, $\eta$ the viscosity of the continuous medium and $\Phi$ the volume fraction of the droplets.

However, the model cannot explain the enhancement in conductivity for high volume fractions in disperse phase. This could be justified due to the importance increase of interactions between droplets.

Kallay and Chittofrati [85] carried out an improvement of this theory, introducing the concept of Born's radius and Stokes' radius, defining the first term as the radius of the aqueous core and the second as the radius of the droplet. If it is considered that the charge is found in the core, we have to use Born's radius. With this change, better results are obtained when the experimental results for the zone prior to percolation are described.

As explained, the percolation phenomenon takes place on increasing temperature or volume fraction of the disperse phase. However, the explanation is not simple. A first theory is based on the existence of a bicontinuous layer so that a channel full of water is formed. Another theory is based on attractive interactions between droplets which form percolation clusters, the charge transport taking place through the tensioactive ions. 
Bhattacharya et al. [86] observed that the conductivity of the $\mathrm{AOT} / \mathrm{C}_{10} /$ water microemulsions increases on increasing temperature and requires a frequency higher than $1 \mathrm{kHz}$. They observed small conductivity values in comparison with those of water, which made think that conduction was governed by interfacial processes. In previous studies on the same reaction system carried out by Kim and Huang [87], it was found that the percolation phenomenon does not depend on frequency for low frequencies $(100 \mathrm{~Hz}, 100$ $\mathrm{KHz}$ ), which would not fit to the idea of a bicontinuous structure. They calculated the necessary time for the production of tensioactive 'hopping' between water droplets at approximately $3 \times 10^{-6}$ seconds. This is the necessary time for $50 \AA$ diffusion [88-90].

If these hoppings were responsible of the droplet conduction, the conduction would only depend on the frequency in the order of $\mathrm{MHz}$, which has been checked. The fact that conductivity might be influenced by temperature variation can be interpreted by assuming that the tensioactive anion has certain probability of leaving the droplet in such a way that, once out of the droplet, might easily suffer a diffusion process. If two free anions of tensioactive are found in the medium, a charge transport will take place. Since the capability of diffusion increases on increasing temperature, the probability of ionizing the tensioactive and the mobility of the ions also increase, leading to a logical enhancement of conductivity.

There is another theory that could explain the percolation phenomenon on the basis of the changes in the microemulsion structure, so that the spherical droplets would transform into cylindrical micelles in the percolation point. However, structural studies of microemulsions do not reveal such change in the percolation zone.

Subsequently, Peyrelasse [91] studied the behavior of the $A O T / C_{11} /$ water system finding as a significant result a delay in percolation on increasing the saline content of the microemulsion. In 1988, Maitra et al. [92] studied the $\mathrm{AOT} / \mathrm{CC}_{8} /$ water microemulsions and the influence of additives in this system, showing that these additives change the percolation threshold because they affect the permeability of the tensioactive film, which demonstrates that the tensioactive film plays an essential role in percolation. The species that increase the permeability of the membrane favor the percolation process, while those that decrease the permeability of the membrane slow down the appearance of this phenomenon.
With this finding, the idea that conduction is due to the diffusion of monomers between droplets is dismissed, another option being preferred. According to this option, the $\mathrm{Na}^{+}$ions are the real charge carriers, going from one droplet to another and therefore, needing an opening of the tensioactive film.

The studies of Lang, Zana et al. [93-94] relate percolation to the matter transport by means of the rate constant, finding that values of $k_{e}$ higher than (12) $\times 10^{9} \mathrm{M}^{1} \mathrm{~s}^{-1}$ are necessary for the percolation to take place. In their study, they observed that the percolation zone moves forward on increasing the attractive interactions between droplets. On the basis of these results, they dismissed the idea that percolation takes place through hoppings of the tensioactive molecules between neighboring droplets in clusters of droplets that are formed in the percolation zone, since this mechanism does not explain the relation between percolation and the rate constant of the matter transport. It is suggested a percolation mechanism through which conductivity could be explained by the counterion movement in the narrow tubes or channels created inside the droplet clusters present in the zone previous to percolation by means of the opening of the tensioactive membrane that surrounds the droplets. The opening of these membranes must be considered a formation of a dimmer droplet with the connected cores. What is needed to happen is an interpenetration of the tensioactive films in the droplet collisions. According to this theory, the tensioactive is important only to the zone prior to percolation, so that percolation is due mainly to counterions.

\section{Effect of Additives on the Electric Percolation}

It is known that some additives, in moderate concentrations, can affect the percolation threshold position. Mathew et al. [92] have established that the additives that provide rigidity to the tensioactive membrane (such as cholesterol Scheme 8) hinder the appearance of the electric percolation phenomenon, while those additives that make the membrane more flexible (such as gramicine Scheme 9) favor it.

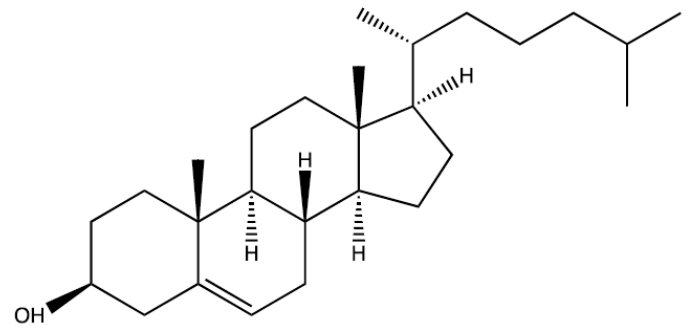

Scheme 8: Cholesterol. 


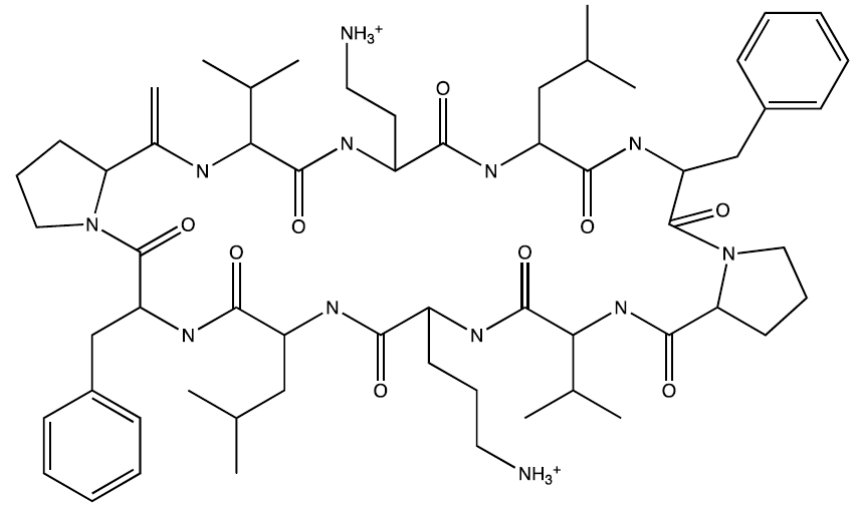

Scheme 9: Gramicine.

These results, together with those found by Lang and Zana [93], indicate that the percolation phenomenon is not associated to the formation of bicontinuous structures in the microemulsion, but it maintains the discrete structure of the droplets. Probably the number of collisions increases suddenly in the vicinity of the percolation point with the formation of clusters of droplets between which, channels are opened to allow the flow of matter between them (vide supra). In this sense, addition of additives to microemulsions will allow modulating the moment of their electric percolation since the modification of the properties of the surfactant film will facilitate or make difficult the opening of the channels that allow the matter exchange between droplets. This can directly result in large or small modifications of the percolation threshold.

Over the last years, comprehensive studies have been carried out on the effect of different additives on the internal dynamic of microemulsions, especially on the effect of additives on the electric percolation phenomenon. In our laboratory, several works [95-98] have been performed in order to analyze the effect of different organic substrates on the electric percolation of $\mathrm{AOT} / \mathrm{iC}_{8} / \mathrm{H}_{2} \mathrm{O}$ microemulsions. Together with these studies, it is also worth to mention the contributions of Moulik et al. on the effect exerted by different additives [99-104] on the electric percolation of $A O T / i C_{8} / \mathrm{H}_{2} \mathrm{O}$, AOT $/ \mathrm{nC}_{7} / \mathrm{H}_{2} \mathrm{O}$ and $\mathrm{AOT} / \mathrm{nC}_{10} / \mathrm{H}_{2} \mathrm{O}$ microemulsions.

Without a doubt, the effect exerted by electrolytes is the most studied case [105]. microdroplets due to the presence of In particular, Cabos and Delord [77] carried out a study on neutron scattering in $\mathrm{AOT} / n \mathrm{C}_{7} / \mathrm{H}_{2} \mathrm{O}$ microemulsions in the presence of $\mathrm{NaCl}$, proposing an increase in the size of the aqueous core of the electrolyte. However, subsequent studies carried out by Lang et al. [76] interpreted results of fluorescence resolved in time, suggesting that the presence of salts reduced the constant of matter exchange between droplets, and produced at the same time a decrease in the value of the aggregation number (i.e., size) of droplets. Likewise, Rouviere et al. [59] observed that the addition of $\mathrm{NaCl}$ and $\mathrm{CaCl}_{2}$ to microemulsions produced a viscosity decrease that could be related to the percolative phenomenon. That was attributed to greater droplet sphericity in the presence of salts, which would imply a decrease of the polar head area.

Salts effect on the system behavior may be considered as a result of the effect of the presence of salts solutions of tensioactives. It is known that salts reduce the dimensions of the effective polar area of surfactants acting as a screen of the electrostatic repulsions [106]. Such reduction causes an increase of the curvature parameter (or critical packing parameter) of the surfactant (vide supra) [107]. The decrease in the effective area of the polar head causes a more noticeable 'trapezoidal' structure (wedge-shaped) of AOT, increasing the negative natural curvature of the tensioactive, i.e., its tendency to give rise to inverse structures. This explains why the presence of salts reduces the water solubilizing capacity of microemulsions [108], since water solubilization -on increasing the droplet size- reduces the negative curvature of the interface, which opposes the normal tendency of the tensioactive. However, focusing on monophasic systems, as Robinson et al. [75] indicates, it is important to discriminate between the natural curvature of the tensioactive and the real one, which is fundamentally determined by reasons of medium composition. In the presence of salts, the area of each headgroup will decrease, but the volume of the disperse phase will remain constant as long as the microemulsion does not break. Basically, a decrease of the area at a constant volume would correspond with a radius increase, as Cabos and Delord [77] observed. However, this enhancement takes place due to a reduction of the tensioactive curvature, which is opposed to the natural tendency of AOT when the saline concentration is increased. If a high quantity of salt is added, the difference between the curvature that the AOT must take and the natural one, will be so remarkable that the monophasic system will be destabilized and will break creating an additional saline aqueous phase that will be eliminated from the w/o microemulsion. Until the elimination takes place, the system will have to keep dimensions similar to those presented in the absence of additive (or at least, with slightly bigger droplets in accordance with the smallest 
superficial area of the heads) since the medium composition is the one, which will limit its geometry [109-110]. However, it remains to be studied the reason why Lang et al. [79] -by using fluorescence with temporal resolution technique- measured lower aggregation numbers in the presence of salts. This fact would indicate an enhancement in the surface/volume relation, and, hence, the superficial area per headgroup. A possible explanation may reside in the idea proposed by Rouviere [59] according to which salts increase the sphericity of the droplets. Even if we have started from the fact that the droplets of the microemulsion are initially spherical, there might be small deformations, with local zones of lower negative curvature. The addition of salts might generate the tendency towards a greater sphericity of the microdroplets and therefore, the same volume of disperse phase might be situated now in a more spherical structure, which gives rise to a lower aggregation number compatible with a smaller surface per polar head.

In any case, the dichotomy between the natural curvature and the real curvature helps to understand the observations regarding the electric percolation phenomenon. The results obtained by García-Río et al. $[89,111-112]$ indicate that the electric charge transport is due to the transfer of $\mathrm{Na}^{+}$ions between droplets. This transport would involve a collision between droplets in order to, at least, give rise to transient dimmers where the tensioactive films would open and the inner contents would mix together. As pointed out by Robinson et al. [73], the opening of the tensioactive films has appreciable activation energy since it implies the creation of local regions of positive curvature. The matter exchange process, and consequently the electric conduction process, would be more difficult. In this sense, the efficiency order of cations -when it comes to hinder this channel opening- corresponds with Hofmeister's series [105] i.e., the radius order of the hydrated cations. The smaller the size of the hydrated cation, the greater the efficiency to associate to the interface is. This will produce a decreasing effect of the area of the mentioned polar headgroup. It is interesting to indicate that a similar series has been observed for the sequence that conducts the cation affinity through the micellar surfaces of 'normal' micelles in water [111-114]. This has been interpreted as evidence of the presence of hydrated counterions in the micellar Stern layer. The results in microemulsions seem to show a parallel case to the one observed in micelles. These results would indicate that the association of cations to the AOT polar heads takes place (at least at $W=22$ ) keeping its hydration water layers. This seems characteristic of the electrostatic attraction of weak electric fields such as those caused by normal ionic micelles. A certain insensitivity of the electric percolation phenomenon to the nature of the salt anion is also coherent with this idea. If the electrostatic repulsion with the AOT headgroups keeps the anions in aqueous regions away from the negatively charged AOT headgroups, the effect of the anion on the tensioactive film (and therefore on its polar area) must be minimum.

The parallel decrease of viscosity in the presence of salts [105] is associated to the decrease of attractive forces between droplets, which is related to their interpenetration (Figure 2). In fact, different theoretical calculations in literature suggest [115-116] that the most important contribution of the attractive interactions occurs in the overlapping zone. As other authors [117] indicate, it is expected that this interpenetrability will be smaller the more rigid the interface is, which seems likely to be achieved by means of a salinity increase.

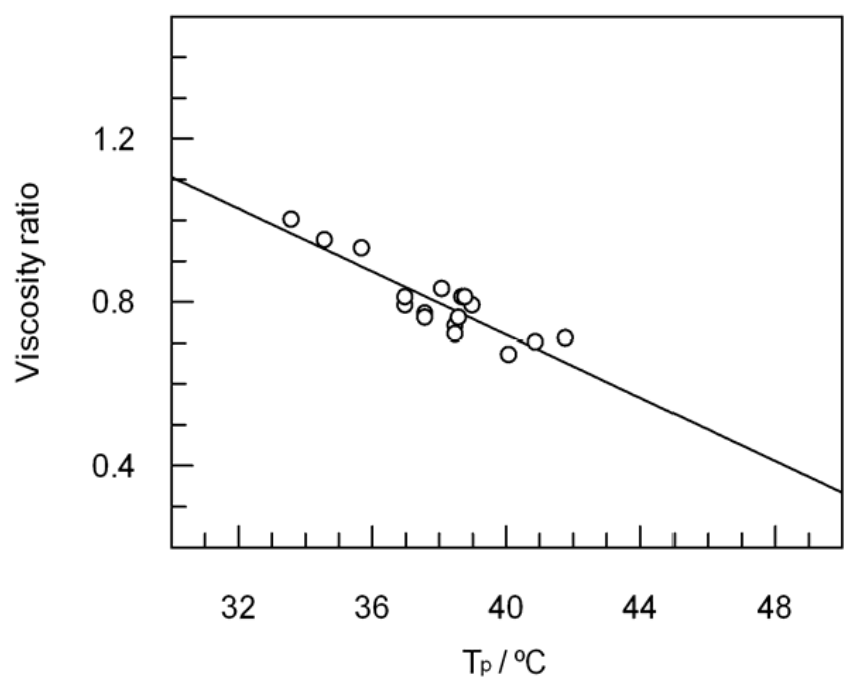

Figure 2: Correlation between $T_{p}$ and relativity viscosity of AOT $/ \mathrm{iC}_{8} / \mathrm{H}_{2} \mathrm{O}$ in presence of different electrolytes (०). $[\mathrm{AOT}]=0.5 \mathrm{M}, W=22.2$, [additive $]=0.04 \mathrm{M}$.

It is interesting and surprising the effect that produces the presence of relatively low salt concentrations, taking into account the great ionic concentration brought by the AOT. For example, in most of the data obtained in our laboratories [111], the concentration of added electrolyte is 60 times smaller to the one already present in the medium and brought by the AOT counterions, but important alterations are observed in the internal dynamics of microemulsions. This fact seems to indicate that such a high ionic 
concentration present in the interior of microemulsions does not produce a saline 'saturation' of the interface, which would keep being sensitive to the addition of salts in salt/AOT molar ratios such as $1 / 62.5$ (this addition might produce a $7^{\circ} \mathrm{C}$ decrease in the percolation point in the case of $\mathrm{CsCl}$ ). According to this, the local alteration of a small portion of the polar headgroups causes important changes on the ability of microemulsions to open channels between droplets.

It is also important to point out that among the electrolytes studied in our laboratory, some belong to the group of 'structure-breaker ions' [118] such as ions with small polarizing power $\left(\mathrm{Rb}^{+}, \mathrm{Cs}^{+}, \mathrm{I}^{-}, \mathrm{ClO}_{4}^{-}\right.$, etc.) that clearly produce an increase in the disorder of the water structure. In particular, the influence of the guanidinium ion has been studied [119] (Scheme 10) which is frequently used as a protein denaturant and hydrocarbon solubilizer (salting-in agent). Likewise, among the list of studied salts, there are also ions known for their capacity of 'structure-maker ions' that on being dissolved in water- increase the level of water-structuring causing an increase of the energetic cost for the destruction of such structures. If the role of added salts on affecting the percolation threshold was related to the properties of the water trapped inside the microemulsion (such as viscosity or water fluidity of the microdroplet) it could be possible to observe opposed effects between the 'structure-breaker ions' and the 'structure-maker ions'. The lack of experimental evidences, or data obtained in our laboratory or in literature about this effect divergence would confirm the fact that the role played by these salts is mainly related to their association at the interface.

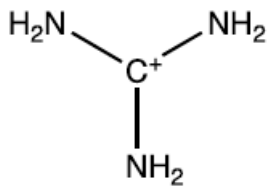

Scheme 10: Guanidinium ion.

In sum, electrolytes increase the negative natural curvature of the tensioactive, increasing the rigidity of the surfactant film whose deformation is obstructed, decreasing the interaction between droplets and increasing the percolation temperature.

Another important group of additives is the small organic molecules. Particularly, it is worth mentioning the results observed for molecules as urea and thiourea [120]. In contrast to electrolytes, these substrates cause a sudden advance in the percolation point so the region of high conductivities may be obtained at unusually low temperatures. In fact, in presence of these additives, it has been obtained electric percolations for a volume fraction of disperse phase much inferior to the theoretical fractions proposed by the geometrical models of percolation (such as the EMT that predicts the percolation for f>0.33) [81], even when corrections are taken into account due to the droplet interaction -EMTDD- (that predicts the percolation for $f>0.15$ ) [121].

The analyzed substrates were several ureas and thioureas whose structures are shown in Scheme 11. The effect exerted by these compounds on the water structure is a controverted issue. Traditionally, the capacity of breaking the water structure of wide range was attributed to ureas, being classified as 'structurebreaker' and thus, their properties were explained as protein denaturant and hydrocarbon solubilizer [106]. However, an important set of experimental data [122121] and the result of theoretical simulations [124-125] indicate a very slight effect of the urea on the water structure. What seems clear is the fact that the substituted ureas function as 'structure-maker', potentializing the structure of the hydrogen bond of water [126]. The efficiency order in promoting the solvent structure seems to increase with the progressive methylation. These studies also indicate that thiourea does not have clear tendencies as 'structure-breaker' or 'structure-maker'. García-Río et al. [105] have covered all the range of ureas and thioureas that dissolve in the aqueous zone or in the interfacial region of the microdroplets. In all cases, the effect has been qualitatively the same: favoring the appearance of the percolation phenomenon, which indicates that its effect is not related to a possible effect of the additives destroying the organized structure of water and favoring the ion transport between droplets. Several aspects strongly suggest that the effect of additives is more related to their interfacial association. For example, the fact that thiourea shows a superior efficiency than urea when speeding up the appearance of the percolation phenomenon -in spite of the fact that both have analogous effects on the water structuremight be related to a greater proportion of thiourea (less polar) in the interfacial region.

The order that these studies show in the case of thioureas is analogous to the observed in ureas, except for the case of tetramethylthiourea, whose effect is smaller than expected, which is justified by the fact that no null solubility of tetramethylthiourea in isooctane causes that part of the additive pass to the 'oil' phase, decreasing its concentration in the microdroplets. 
<smiles>NC(N)=O</smiles>

Urea<smiles>CNC(N)=O</smiles>

Methylurea<smiles>CNC(=O)NC</smiles>

1,3-Dimethylurea<smiles>CN(C)C(N)=O</smiles>

1,1-Dimethylurea<smiles>CNC(=O)C(C)C</smiles>

Trimethylurea<smiles>CC(C)C(=O)N(C)C</smiles>

Tetramethylurea<smiles>NC(N)=S</smiles>

Thiourea<smiles>CNC(N)=S</smiles>

Methylthiourea<smiles>CN(C)C(N)=S</smiles>

1,1-Dimethylthiourea<smiles>CNC(=S)NC</smiles>

1,3-Dimethylthiourea<smiles>CNC(=S)C(C)C</smiles>

Trimethylthiourea<smiles>CC(C)C(=S)N(C)C</smiles>

Tetramethylthiourea

\section{Scheme 11:}

Additionally or alternatively, the greater size of this additive might sterically hinder its association to the interfacial layer with regard to its analogues. In any case, in view of the results of these authors, it seems clear that these additives operate by association to the micellar interface, as suggested also by the studies carried out on the effect of ureas and substituted urea's in normal micelles and vesicles and by the conclusions drawn by Polito et al. [127] in their studies on urea in $\mathrm{AOT} / n \mathrm{C}_{7} /$ water microemulsions. If ureas associate with the AOT polar heads, they can produce an increase in the effective area of these heads, originating a decrease in the curvature parameter of the tensioactive. This decrease will facilitate -following the opposite reasoning to the one used in the case of saltsthe fusion between droplets on decreasing the natural tendency of AOT towards the formation of inverse structures. In addition, the association of the additive to the heads might cause a major degree of disorder in the interfacial zone, decreasing the rigidity of the AOT film, which would facilitate its deformability. Regarding this point, it is interesting to question whether the role of ureas and thioureas in the interfacial region is substituting the water molecules or not. The study by Kevan et al. [128] on the effect of various ureas on frozen vesicles seem to suggest that the addition of moderate concentrations of urea or dimethylurea 'opens' the interface and even facilitates a higher water penetration into the micellar structure. As in the case of electrolytes [89,111-112] a linear relationship between relative viscosity of microemulsions and percolation temperature has been found (Figure 3), and the viscosity behaviour brought about by ureas and thioureas seems to be due to a greater extent of interdroplet attraction, which may be related to the decrease in interfacial rigidity (vide supra).

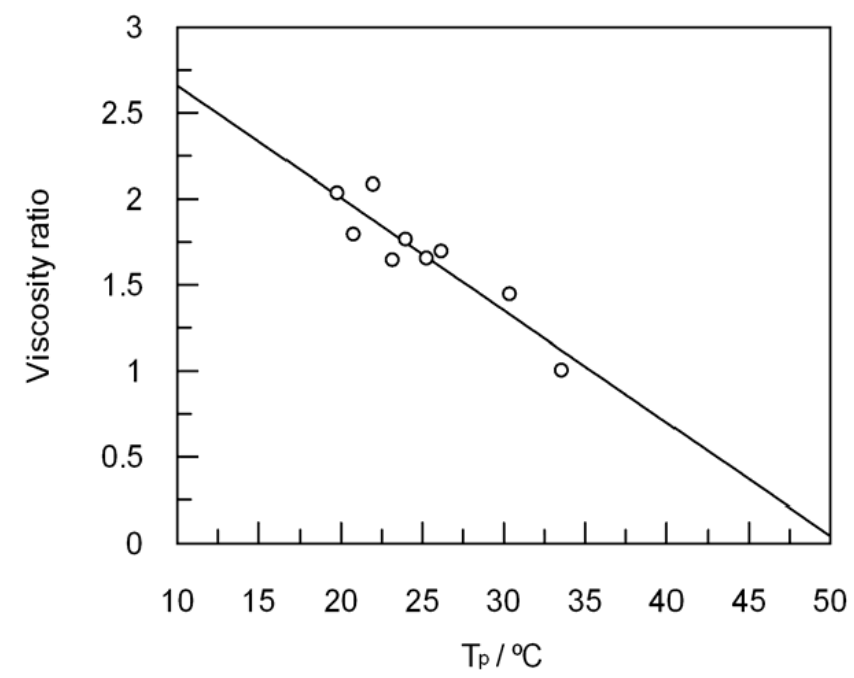

Figure 3: Correlation between $T_{p}$ and relativity viscosity of $\mathrm{AOT} / \mathrm{iC} \mathrm{C}_{8} / \mathrm{H}_{2} \mathrm{O}$ in presence of ureas and thioureas $(0)$. $[\mathrm{AOT}]=0.5 \mathrm{M}, W=22.2$, [additive] $=0.2 \mathrm{M}$.

According to other authors [129] for the case of alkanol we find that $K, K_{d}$ and $K^{\prime}$ are affected by temperature, and also by the length of the carbon chain 
<smiles>CC(C)(C)CC(C)(C)N</smiles><smiles>CNC(C)(C)CC(C)(C)C</smiles><smiles>CN(C)C(C)(C)CC(C)(C)C</smiles>

Scheme 12: Alkyl amines.

$$
K=\frac{n_{a}^{o}}{n_{o}}
$$

where $n^{\circ}$ is the number of alkanol moles in the oil phase, and $n_{0}$ is the total number of moles in oil.

$$
K_{d}=\frac{n_{a}^{i} n_{o}}{n_{s}^{i} n_{a}^{o}}
$$

where $n_{a}$ would be the total number of alkanol moles and the $i$ and $o$ superscripts correspond to the number of alkanol moles in the interface and the oil phase; we define a new term $n_{s}^{i}$ for the number of surfactant moles in the oil phase. Thus, we can also define it like:

$$
K^{\prime}=K K_{d}=\frac{n_{a}^{i}}{n_{s}^{i}}
$$

Nevertheless, at high additive concentrations, it can replace water molecules of the interface, being directly involved in the solvation of the headgroups. This aspect can be elucidated by analyzing the effect of 1,3dimethylurea on the signal of ${ }^{1} \mathrm{H}-\mathrm{NMR}$ of the water protons of the microemulsion, a signal that is very sensitive to the proportions of free water of the microemulsion. These NMR data indicate that the chemical movements of water (that strongly change with $W$ parameter) are however, independent of the added concentrations of additive. This suggests that on associating to the interface, the additive does not cause any remarkable alteration in the relative number of water molecules present in each different state within the microemulsion, since it does not produce a significant movement of the water molecules of the interface.
Analogous results have been obtained for other organic substrates such as amines [130], alcohols [131] and amides [88].

The various effects of $n$-alkylamines on the electric percolation of $\mathrm{AOT} / \mathrm{iC}_{8} / \mathrm{H}_{2} \mathrm{O}$ microemulsions studied by García-Río et al. [132-133] are especially relevant for our study (Scheme 12). These authors confirm that the presence of alkylamines of linear chain causes an enhancement in the percolation temperature, fundamentally attributed to the association of the amine to the interface, playing a role similar to that of a cosurfactant. The increase is also attributed to the different solubility of the amine between the three pseudophases of the system. In this case, satisfactory correlations have been found between the percolation temperature of the different alkylamines and the carbon number in the alkyl chain, and also with the values of

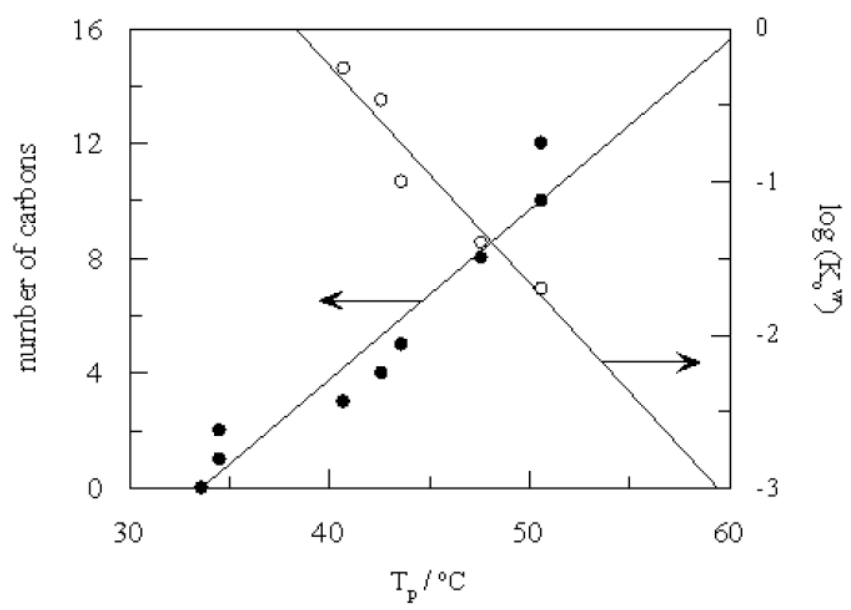

Figure 4: Correlation between $T_{p}$ and the carbon number of the hydrocarbon chain of $n$-alkylamines ( $\circ)$ and correlation between $T_{p}$ and the kinetically obtained partition coefficient of the amines between water and oil [139] $(\bullet)$. [AOT] $=0.5 \mathrm{M}$, $W=22.2$, [additive] $=0.01 \mathrm{M}$. 
the distribution constant of the amines between the aqueous and organic phase $\left(K_{o}{ }^{w}\right)$ and with their $p K_{a}$ values. The above-mentioned correlations are presented in Figures $\mathbf{4}$ and $\mathbf{5}$.

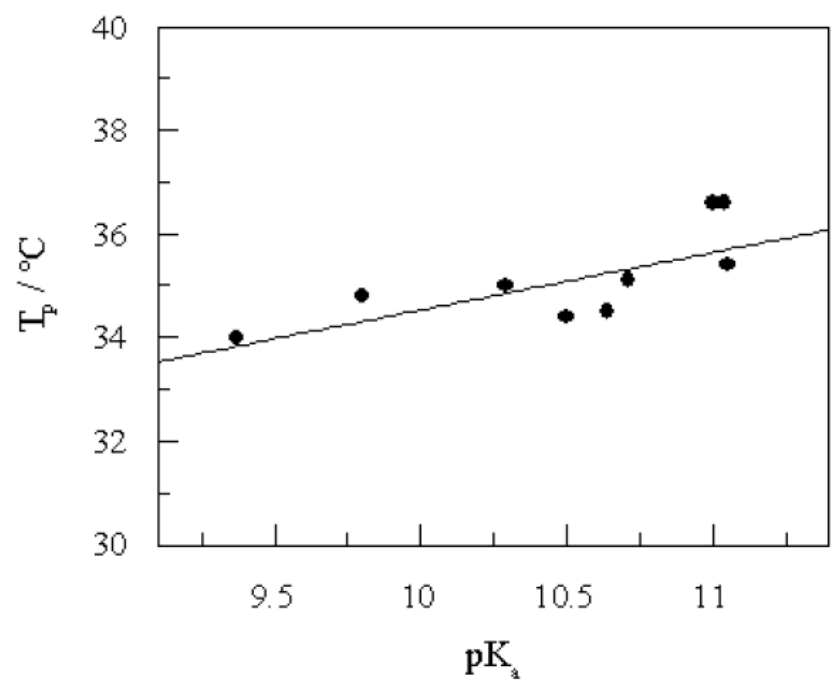

Figure 5: Correlation between percolation temperature and $p K_{a}$ of the amines. [AOT] $=0.5 \mathrm{M}, W=22.2$, [additive] $=0.01 \mathrm{M}$.

A more surprising result is the one observed for the addition of alkylsulphonates and alkylsulfates [134] with hydrocarbon chains comprised between $\mathrm{C}_{3}$ and $\mathrm{C}_{18}$ to $\mathrm{AOT} / \mathrm{i} \mathrm{C}_{8} / \mathrm{H}_{2} \mathrm{O}$ microemulsions, keeping constant the ratio between the alkylsulphonate and AOT concentrations $(\rho=0.01)$ (Scheme 13). The observed behaviour corresponds with a bimodal tendency (Figure 6) where a group of alkylsulphonates delays the percolation temperature $\left(\mathrm{C}_{3}-\mathrm{C}_{5}\right)$, so as to later speed up the process $\left(\mathrm{C}_{6}-\mathrm{C}_{18}\right)$. However, the speeding up of the percolation threshold does not correspond with the increase in the chain length (does not correlate with the $c m c$ of these surface agents in aqueous solution and therefore, does not correlate with the enhancement of the hydrophobic interactions on increasing the carbon number in the tail of the amphiphile) since this decrease reaches a minimum for $\mathrm{C}_{8}$, in order to present a behavior analogous to the one observed in the case of alkylsulphates in the range $\mathrm{C}_{10^{-}}$ $\mathrm{C}_{18}$.
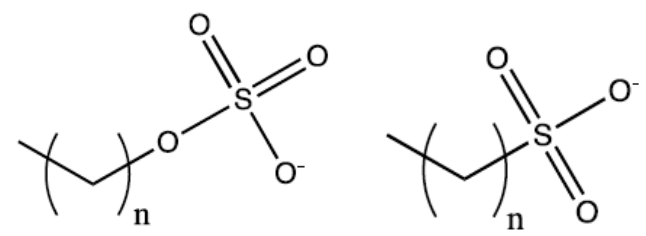

Scheme 13: n-alkyl sulfates \& n-alkyl sulfonates.

A similar behavior has been found in the bibliography when analyzing the influence of alkylsulphonates on the percolation of non-ionic microemulsions [135-137], and n-alkyl carboxilic acids [138].

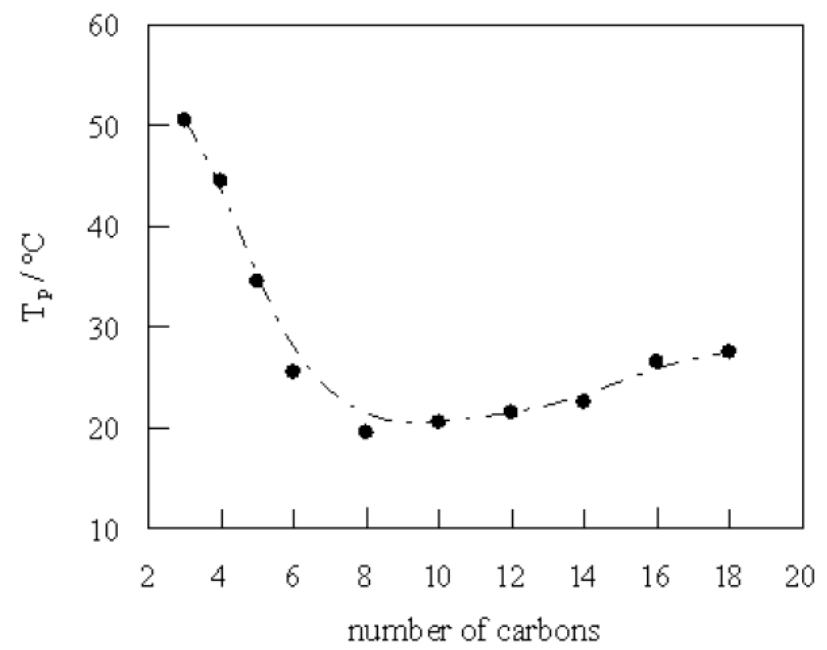

Figure 6: Change of the percolation temperature for $\mathrm{AOT} / i \mathrm{C}_{8} /$ water microemulsions in the presence of alkylsulphonates depending on the chain length of the surfactant. $W=22.2, \rho=0.01,[\mathrm{AOT}]=0.5 \mathrm{M}$.

In order to rationalize this behavior, the authors have taken into account the dual nature that the alkylsulphonates might present. They are sodium salts, but, on the other hand, they have substances that -on having a hydrocarbon tail with a polar head- have a more or less marked behavior as amphiphiles. In this sense, for short chain lengths, the predominant effect on observing the addition of these substrates is the corresponding to an increase in the $\mathrm{Na}^{+}$concentration within the aqueous microdroplet. Its effect on the electric percolation would be justified by the decrease of the effective area of the surfactant head due to the screening of the electrostatic repulsions between the charged groups of the tensioactives [139]. Thus, it could be considered that for short-chain lengths, the main role of these additives is that of becoming an extra source of cations in the aqueous microdroplet, and therefore, their behavior would be the equivalent to the addition of an electrolyte (vide supra). For longchain tensioactives the main effect would be the inclusion of a different structural unit in the interface that would modify its packing (Scheme 14). This packing change would allow regions within the interface where the formation of positive curvatures would be more favorable. It is obvious that this circumstance could be helpful in the formation of channels for the matter exchange between droplets and therefore, would facilitate the percolation process.

It is necessary to point out that for the substrates where their amphiphilic nature seems to dominate, the 
percolation temperature increases up to the value of $\mathrm{C}_{8}$, moment when there is no significant effect on the percolation temperature (range of chain lengths comprised between 8 and 18). In fact, the authors observe a difference of $31^{\circ} \mathrm{C}$ between $\mathrm{C}_{3}$ and $\mathrm{C}_{8}$, whereas between $\mathrm{C}_{8}$ and $\mathrm{C}_{18}$ the variation of the percolation temperature is barely $8^{\circ} \mathrm{C}$, and the observed difference between $\mathrm{C}_{16}$ and $\mathrm{C}_{18}$ is barely $0.5^{\circ} \mathrm{C}$ (a value that could even be attributed to an experimental error).

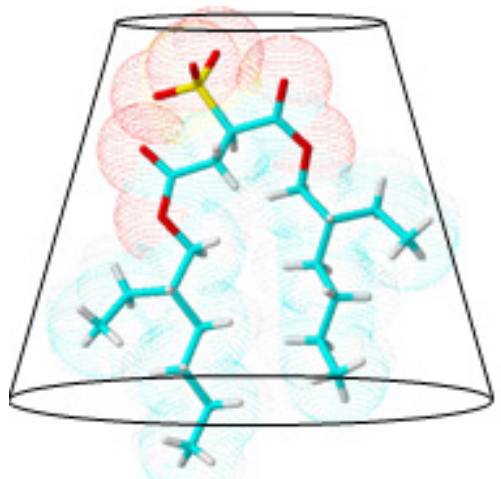

a

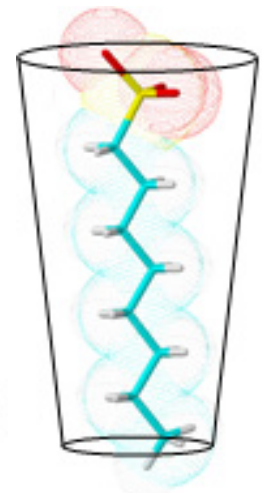

b
Scheme 14: a. Packing parameter favoring inverse structures. b. Packing parameter favoring direct structures.

The obtaining of a minimum for $\mathrm{C}_{8}$ is attributed by the authors to the fact that this molecule presents a chain length similar to the AOT chain, because of which the latter would be the one that would incorporate more adequately to the surfactant film. For longer chain lengths (whose length is greater than the AOT chain) they do not adapt adequately to the surfactant film, which causes a process of looping of the surfactant chain, similar to the one observed for normal micelles, where there have been found experimental evidences that suggest that there is a certain degree of contact between the water and the hydrocarbon tails [140]. In this sense, the differences in surfactants ${ }^{1} \mathrm{H}-\mathrm{NMR}$, such as $\mathrm{LTABr}, \mathrm{TTABr}$ and $\mathrm{CTABr}$, that have 12, 14 and 16 carbon atoms respectively, show that the most affected protons are those situated in the headgroup (in $\alpha$ position to the above mentioned group) and in the terminal carbon [141]. Therefore, this looping phenomenon in the surfactant chains would cause the reduction of the impact of inclusion of the alkylsulphate in the AOT film (see Scheme 15). Such reduction would increase with the increase of its hydrocarbon tail, since this increase in the chain length results in an increase of the possibility of the looping phenomenon to take place.

The micellization behaviour of cetylammonium bromide (CTABr), its derivatives, the analogous trihydroxyethylated headgroups and the formation of w/o microemulsions has been studied by means of thermodynamic and structural considerations by authors like Maitra et al. [92], solving the efficiency of surfactants with regard to the dispersion into oil and the level of cosurfactant concentration in the $o / w$ interface.

In the case of n-alkyl carboxylic acids [138], the effect of the addition of different n-alkyl acids (from 1 to 24 carbons in the hydrocarbon chain Scheme 16) upon the percolative phenomenon in $\mathrm{AOT} / \mathrm{iC}_{8} / \mathrm{H}_{2} \mathrm{O}$ microemulsions was studied in our laboratory. In general, at low organic acid concentrations, the percolation temperature increases on increasing the additive concentration. Such a behavior could be explained considering that the amount of $\mathrm{H}^{+}$in the aqueous core of the droplet in the microemulsion is increased by the presence of these additives. The effect of the anion could be neglected, as occurs in the literature for similar concentrations of the different

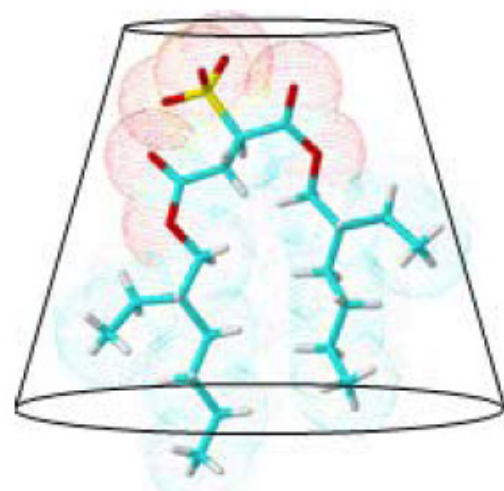

a

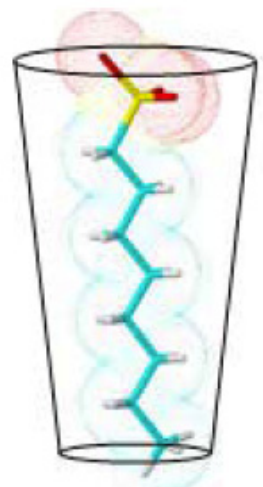

b

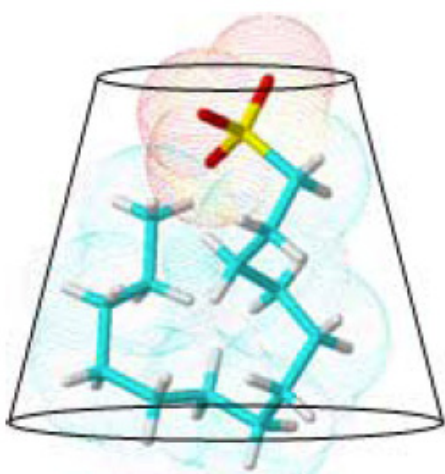

C

Scheme 15: a. Geometry favoring inverse structures. b. Geometry favoring direct structures. c. Geometry favoring inverse structures. 
sodium halides, where the effect upon percolation temperature is related to the concentration of cations and independent of the nature of the anion [105,112]. So, it seems that indeed increasing the presence of cations in the nearby of the headgroup of the tensioactive also means an increase in the screening effect exerted on the electric charge of the headgroup (vide supra). A similar explanation can be considered for hydrochloric acid $\left(\mathrm{H}^{+}\right.$partially replace $\left.\mathrm{Na}^{+}\right)$, where a linear relationship of the $\left[\mathrm{H}^{+}\right]$and the percolation temperature have been observed (at least with values of $\mathrm{W}=22.2$ and $[\mathrm{AOT}]=0.5 \mathrm{M}$ [89]), a relationship was never found for $n$-alkyl acids. An increase in concentration for $n$-alkyl acids increases percolation temperature until reach a maximum, and then, it begins to decrease, reaching values even lower than the ones observed with no additive at all. Such behaviour was found in the case of the n-alkyl acids of shorter chain length, but from the valeric acid and more length $\left(\mathrm{C}_{5^{-}}\right.$ $\mathrm{C}_{24}$ ), only the initial increase in the percolation temperature has been reported, and then it levels off [138].

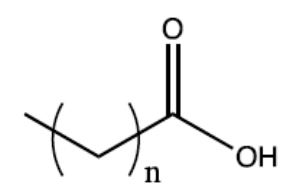

Scheme 16: n-alkyl acids.

This stabilization may be caused by the organic anions influencing the microemulsion. To evaluate this effect, the influence of increasing quantities of $\mathrm{Na}^{+}$ (added as $\mathrm{NaCl}$ ) [111] and the influence of increasing quantities of $\mathrm{Na}^{+}$(added as sodium formate) [138] were compared. The addition of increasing amounts of $\mathrm{NaCl}$ carries increased percolation threshold, something also occurs with the addition of sodium formate, which demonstrated to exert a higher effect on the percolation than the $\mathrm{NaCl}$, probably because $\mathrm{H}^{+}$is better than $\mathrm{Na}^{+}$ at stabilizing the tensioactive headgroup charge. As it has been mentioned before, effect of monovalent positive ions respect Hofmeister's series, so adding an extra of $\mathrm{H}^{+}$has a deeper effect that an extra amount of $\mathrm{Na}^{+}$. Also, the presence of notable quantities of unprotonated organic compounds in the microemulsion must be considered, as long as they would exert a similar effect to other organic substances $[88,120$, 132]. These compounds solubilize in the tensioactive film, something that implies the integration in the film of those molecules with a critical packing parameter lower than $1 / 3$. This way, critical packing parameter in the area is reduced and a positive curvature of the film is facilitated. In other words, percolation threshold and percolation temperature is decreased.

On the other hand, n-alkyl acids may have a combination of both antagonistic effects on the percolation threshold: the increasing caused by the supply of $\mathrm{H}^{+}$, and the decreasing ought to the organic, non-dissociated substrates. The influence upon the electrical percolation of alkyl-acids as additive in AOT microemulsions yields an evidence of the inclusion of these molecules in the AOT film (both as protonated and as unprotonated molecules), and this inclusion will modify the internal dynamics of the AOT-based microemulsions. As long as microemulsions are often utilized as chemical nanoreactors (which is specifically true for AOT microemulsions) changes in the stiffness of the tensioactive film may be of importance [142-146]. Also, some applications for these systems have been reported related to lipase-catalysed processes [147149].

In conclusion, it seems that additives affect properties of AOT-microemulsions because they, or their related ions, associate with the surfactant interface and interfere with it. Increase in the percolation temperature and reduction in viscosity appear to be due essentially to the decreasing in the effective area of the polar heads of the surfactant and increasing the rigidity of the interface; also, decrement in the percolation temperature, and also in viscosity, is mainly owed to an increase in the headgroup area of the tensioactive and a decrease in the stiffness of interfaces [105]. It must be remarked that these additives effect not only in the facilitation of the transport phenomena that allow electric percolation to happen, but on the reaction rates in processes that occur within microemulsions.

\section{ACKNOWLEGMENTS}

Authors are grateful to the European Union for provision of FEDER funds. A. Cid acknowledges the post-doctoral grant SFRH/BPD/78849/2011, given by the Portuguese Foundation for Science and Technology (Fundação para a ciência e tecnologia (MEC-FCT)) from Portugal.

\section{REFERENCES}

[1] Summers $M$ and Eastoe J. Applications of polymerizable surfactants. Adv Coll Interface Sci 2003; 110: 137-52. http://dx.doi.org/10.1016/S0001-8686(02)00058-1

[2] Fendler JH. Atomic and molecular clusters in membrane chemistry. Chem Rev 1987; 87: 877-99. http://dx.doi.org/10.1021/cr00081a002 
[3] Fendler JH. Interactions and Kinetics in Membrane Mimetic. Ann Rev Phys Chem 1984; 35: 137-57.

http://dx.doi.org/10.1146/annurev.pc.35.100184.001033

[4] Fendler JH. Reactiviy control in membrane mimetic system. Pure Appl Chem 1982; 54: 1809-81. http://dx.doi.org/10.1351/pac198254101809

[5] Fendler JH. Microemulsions, micelles, and vesicles as media for membrane mimetic photochemistry. J Phys. Chem. 1980; 84: 1485-91.

http://dx.doi.org/10.1021/j100449a012

[6] Langevin D, Meunier J and Cazabat AM. Microemulsions. La Recherche. 1985; 16: 720-28.

[7] Moss RA, Bizzigotti GO and Ihara Y. Biomimetic chemistry of functional vesicles and micelles. Stud Org Chem 1983; 13: 189-205.

[8] Breslow R. Biomimetic Chemistry in Oriented Systems. Israel J Chem 1979; 18: 187-91. http://dx.doi.org/10.1002/ijch.197900024

[9] Garti N and Aserin A. Microemulsions for solubilization and delivery of nutraceuticals and drugs. Drugs and Pharm Sci 2006; 158: 345-428.

[10] Salager JL, Anton RE, Sabatini DA, Harwell JH, Acosta EJ and Tolosa LI. Enhancing solubilization in microemulsionsState of the art and current trends. J Surf Deterg 2005; 8: 321.

http://dx.doi.org/10.1007/s11743-005-0328-4

[11] Eastoe $\mathrm{J}$ and Bumajdad A. Mixed surfactant microemulsions. Rec Res Dev Phys Chem 2000; 4: 337-50.

[12] García-Río L, Mejuto JC and Pérez-Lorenzo M. First evidence of simultaneous different kinetic behaviors at the interface and he continuous medium of w/o microemulsions. J Phys Chem B 2006; 110: 812-9. http://dx.doi.org/10.1021/jp055270o

[13] Shervani $Z$ and Ikushima $Y$. The promotion of hydrolysis of acetylsalicylic acid in AOT/near-critical propane microemulsion. Chem Comm 2001; 23: 2506-7.

http://dx.doi.org/10.1039/b103695p

[14] Pokhriyal NK, Sanghvi PG, Shah DO and Devi S. Kinetics and behavior of copolymerization in emulsion and microemulsion systems. Langmuir 2000; 16: 5864-70.

http://dx.doi.org/10.1021/la991139u

[15] Holmes JD, Steytler DC, Rees GD and Robinson BH. Bioconversions in a water-in- $\mathrm{CO}_{2}$ microemilsion. Langmuir 1998; 14: 6371-6. http://dx.doi.org/10.1021/la9806956

[16] Kishida M, Umakoshi K, Ishiyama J, Nadata $\mathrm{H}$ and Wakabayashi K. Hydrogenation of carbon dioxide over metal catalysts prepared using microemulsion. Cat Today 1996; 29: 355-9. http://dx.doi.org/10.1016/0920-5861(95)00304-5

[17] Das ML, Bhattacharya PK and Moulik SP. Reaction kinetics in microemulsion medium. 1. Inversion of cane sugar in quaternary system of microemulsion containing water/Triton X-100/1-butanol/(cholesteryl benzoate + n-heptane). Langmuir 1990; 6: 1591-5.

http://dx.doi.org/10.1021/la00100a011

[18] Da Rocha Pereira R, Zanette D and Nome F. Application of the pseudophase ion-exchange model to kinetics in microemulsions of anionic detergents. J Phys Chem 1990; 94: 356-61.

http://dx.doi.org/10.1021/j100364a061

[19] Zilman AG and Safran SA. Thermodynamics and structure of self-assembled networks. Phys. Rev. E 2002; 66: 051107/1051107/28.

http://dx.doi.org/10.1103/PhysRevE.66.051107

[20] Dvolaitzky M, Guyot M, Lagües M, Le Pesant JP, Ober R, Sauterey C, Taupin C. A structural description of liquid particle dispersions: Ultracentrifugation and small angle neutron scattering studies of microemulsions. J Chem Phys 1978; 69: 3279-88.

http://dx.doi.org/10.1063/1.436979

[21] Pileni MP. Structure and reactivity in Reverse Micelles. Amsterdam: Elsevier; 1989.

[22] a) Winsor PA. Hydrotropy, solubilization, and related emulsification processes. Part I. Trans Faraday Soc 2 1948; 44: 376-82. b) Formariz TP, Urban MCC, Silva Jr AA, Gremião MPD, Oliveira G. Microemulsões e fases líquidas cristalinas como sistemas de liberação de fármacos. Braz J Pharm Sci 2005; 41: 301-13.

[23] Bauer A, Woelki S and Kohler HH. Rod formation of ionic surfactants: Electrostatic and conformational energies. J Phys Chem B 2004; 108: 2028-37. http://dx.doi.org/10.1021/jp036088v

[24] Dickson JL, Psathas PA, Salinas B, et al. Formation and growth of water-in- $\mathrm{CO}_{2}$ miniemulsions. Langmuir 2003; 19: 4895-04. http://dx.doi.org/10.1021/la0268810

[25] Hait SK and Moulik SP. Interfacial Composition and Thermodynamics of Formation of Water/lsopropyl Myristate Water-in-Oil Microemulsions Stabilized by Butan-1-ol and Surfactants Like Cetyl Pyridinium Chloride, Cetyl Trimethyl Ammonium Bromide, and Sodium Dodecyl Sulfate. Langmuir 2002; 18: 6736-44. http://dx.doi.org/10.1021/la011504t

[26] Vollmer J, Vollmer D and Strey R. Oscillating phase separation in microemulsions II: Description by bending free energy. J Chem Phys 1997; 107: 3627-33. http://dx.doi.org/10.1063/1.474720

[27] Paul S and Moulik SP. Physicochemical studies on microemulsions. IV-A comprehensive estimation of the energetics. Indian J Chem 1995; 34: 931-7.

[28] Nagaranjan R and Ruckenstien E. Theory of surfactant selfassembly: a predictive molecular thermodynamic approach. Langmuir 1991; 7: 2934-69.

http://dx.doi.org/10.1021/la00060a012

[29] Rosano HL and Lyons GB. Free energy, enthalpy, and entropy changes during the formation of a nhexadecane/potassium stearate/water/1-pentanol microemulsion system. J Phys Chem 1985; 89: 363-5. http://dx.doi.org/10.1021/j100248a036

[30] Stecker MM and Benedek GB. Theory of multicomponent micelles and microemulsions. J Phys Chem 1984; 88: 651944.

http://dx.doi.org/10.1021/j150670a014

[31] Kertes AS and Lai WC. Thermodynamics of microemulsion systems. II. Enthalpies of solution in aqueous sodium chloride + 2-propanol systems. J Coll Int Sci 1980; 76: 48-54. http://dx.doi.org/10.1016/0021-9797(80)90269-6

[32] Ruckenstein E. On the thermodynamic stability of microemulsions. J Coll Int Sci 1978; 66: 369-71. http://dx.doi.org/10.1016/0021-9797(78)90320-X

[33] Ruckenstein E and Chi JC. Stability of microemulsions. J Chem Soc Faraday Trans 2 1975; 71: 1690-707. http://dx.doi.org/10.1039/f29757101690

[34] Tanford C. Micelle shape and size. J Phys Chem 1972; 76 : 3020-4. http://dx.doi.org/10.1021/j100665a018

[35] Mitchell DJ and Ninham BW. Micelles, vesicles and microemulsions. J Chem Soc Faraday Trans 2 1981; 7: 60129. http://dx.doi.org/10.1039/f29817700601

[36] Israelachvili JN, Mitchell DJ and Ninhan BW. Theory of selfassembly of hydrocarbon amphiphiles into micelles and bilayers. J Chem Soc Faraday Trans 2 1976; 72: 1525-68. http://dx.doi.org/10.1039/f29767201525 
[37] Sager WFC and Blokhuis EM. Curvature energy for droplet dimerization and aggregation in microemulsions. Prog Coll Pol Sci 1998; 110: 258-62. http://dx.doi.org/10.1007/BFb0118088

[38] Ruckenstein $E$ and Krishnan $R$. The equilibrium radius and the domain of existence of microemulsions. Coll Int Sci 1980; 76: $188-200$.

http://dx.doi.org/10.1016/0021-9797(80)90285-4

[39] Ruckenstein $E$ and Krishnan $R$. The equilibrium radius of microemulsions formed with ionic surfactants. J Coll Int Sci 1980; 75: 476-92.

http://dx.doi.org/10.1016/0021-9797(80)90472-5

[40] Doerfler HD. Structure formation in amphiphilic multicomponent systems. Tens Surf Det 1994; 31: 29-35.

[41] Nagarajan R. Theory of micelle formation: quantitative approach to preceding micellar properties from surfactant molecular structure. Surf Sci Ser 1997; 70: 1-81.

[42] Cazabat AM, Chatenay D, Langevin D and Meunier J. Percolation and critical points in microemulsions. J Chem Soc Faraday Discuss 1982; 76: 291-3. http://dx.doi.org/10.1039/dc9837600291

[43] Kaler EW, Davis HT and Scriven LE. Toward understanding microemulsion microstructure. II. J Chem Phys 1983; 79: 5685-92.

http://dx.doi.org/10.1063/1.445689

[44] Steinchen A, Sanfeld A and Devillez C. Structure and phase inversion of microemulsions in correlation with low interfacial tension. Role of alcohol-role of salinity. J. Chim Phys. et Phys-Chim. Biol. 1980; 77: 229-33.

[45] Ramachandran C, Vijayan S and Shah DO. Effect of salt structure of middle phase microemulsions using the spinlabel technique. J Phys Chem 1980; 84: 1561-7. http://dx.doi.org/10.1021/j100449a025

[46] Bansal VK, Shah DO and O'Connell JP. Influence of alkyl chain length compatibility on microemulsion structure and solubilization. J Coll Int Sci 1980; 75: 462-75. http://dx.doi.org/10.1016/0021-9797(80)90471-3

[47] Kumar $C$ and Balasubramanian D. Structural features of water-in-oil microemulsions. J Phys Chem 1980; 84: 1895-9. http://dx.doi.org/10.1021/j100452a006

[48] Oakenfull D. Constraints of molecular packing on the size and stability of microemulsion droplets. J Chem Soc Faraday Trans 1: Phys Chem in Cond Phases 1980; 76: 1875-86. http://dx.doi.org/10.1039/f19807601875

[49] Chen SJ, Evans DF and Ninham BW. Properties and structure of tree-component ionic microemulsions. J Phys Chem 1984; 88: 1631-4.

http://dx.doi.org/10.1021/j150652a038

[50] Beaglehole D, Clarkson MT, Upton A. Structure of the microemulsion/oil/water interfaces. J Coll and Int Sci 1984; 101: $330-5$ http://dx.doi.org/10.1016/0021-9797(84)90042-0

[51] Sjöblom E, Waernheim T, Henriksson $U$ and Stenius P. The importance of the cosurfactant and the oil for the properties of microemulsions. Tens Deterg 1984; 21: 303-6.

[52] Fletcher PDI, Galal MF, Robinson BH. Structural study of aerosol-OT-stabilized microemulsions of glycerol dispersed in n-heptane. J Chem Soc Faraday Trans 1: Phys Chem in Cond Phases 1984; 80: 3307-14. http://dx.doi.org/10.1039/f19848003307

[53] Roux D, Bellocq AM and Bothorel P. Effect of the molecular structure of components on micellar interactions in microemulsions. Prog Coll Polym Sci 1984; 69: 1-11.

[54] Blum FD, Pickup S, Ninham B, Chen SJ and Evans DF. Struture and dynamics in three-component microemulsions. J Phys Chem 1985; 89: 711-3.

http://dx.doi.org/10.1021/j100250a030
[55] Velázquez MM and González-Blanco C. Polymer effects on the structure and properties of w/o microemulsions. Rec Dev Coll Int Res 2003; 1: 347-359.

[56] Donescu D, Fusulan L, Vasilescu M, Donescu A, Chiraleu F and Petcu C. The influence of monomers upon microemulsions with short chain cosurfactant. J Disp Sci Technol 2001; 22: 231-44.

http://dx.doi.org/10.1081/DIS-100105210

[57] Ozawa K, Olsson U and Kunieda H. Oil-induced structural change in nonionic microemulsions. J Dispersion Sci Technol 2001; 22: 119-24.

http://dx.doi.org/10.1081/DIS-100102687

[58] Rouviere J, Couret JM, Lindheimer M, Dejardin JL and Marony R. Structure of the AOT reverse aggregates. I. Shape and size of AOT micelles. J Chim Phys-Chim Biol 1979; 76: 289-296.

[59] Rouviere J, Couret JM, Lindheimer A, Lindheimer $M$ and Brun B. Structure of AOT reverse aggregates. II. Salt effects upon AOT reverse micelles. J Chim Phys-Chim Biol 1979; 76: 297-301.

[60] Schulman JH, Stoeckenius W and Prince L. Mechanism of formation and structure of micro emulsions by electron microscopy. J Phys Chem 1959; 63: 1677-80.

http://dx.doi.org/10.1021/j150580a027

[61] Talmon $Y$ and Prager S. Statistical thermodynamics of phase equilibriums in microemulsions. J Phys Chem 1978; 69: 2984-91.

http://dx.doi.org/10.1063/1.437016

[62] Taupin C. New ideas for microemulsions structure: the Talmon-Prager and Gennes models. Struct Dyn Mol Syst 1986; 2: 195-208.

http://dx.doi.org/10.1007/978-94-009-4662-0 10

[63] Dvolaitzkay M, Guyot M, Langues M, Le Pesant JP, Ober R and Taupin C. A structural description of liquid particle dispersions: Ultracentrifugation and small angle neutron scattering studies of microemulsions. J Chem Phys 1978; 69: 3279-88.

http://dx.doi.org/10.1063/1.436979

[64] Drifford M, Tabony $J$ and De Geyer A. Structure of a microemulsion in the critical region: neutron small-angle scattering results. Chem Phys Lett 1983; 96: 119-25.

http://dx.doi.org/10.1016/0009-2614(83)80129-8

[65] Shukla $A$ and Neubert $\mathrm{RHH}$. Investigation of W/O microemulsion droplets by contrast variation light scattering. Pramana 2005; 65: 1097-108. http://dx.doi.org/10.1007/BF02705284

[66] Capuzzi G, Pini F, Gambi CMC, Monduzzi M, Baglioni P and Teixeira J. Small-Angle Neutron Scattering of $\mathrm{Ca}(\mathrm{AOT})_{2} / \mathrm{D}_{2} \mathrm{O} /$ Decane Microemulsions. Langmuir 1997; 13: 6927-30.

http://dx.doi.org/10.1021/la970561c

[67] Caboi F, Capuzzi G, Baglioni $P$ and Monduzzi M. Microstructure of AOT/Water/Decane w/o Microemulsions. J Phys Chem 1997; 101: 10205-12. http://dx.doi.org/10.1021/jp971274k

[68] Eriksson JC and Ljunggren S. General conditions governing the formation of cylindrical W/O microemulsion aggregates. J Coll Interf Sci 1991; 145 224-34.

http://dx.doi.org/10.1016/0021-9797(91)90114-N

[69] Leung $R$ and Shah DO. Solubilization and phase equilibria of water-in-oil microemulsions. I. Effects of spontaneous curvature and elasticity of interfacial films. J Coll Interf Sci 1986; 120: 320-9. http://dx.doi.org/10.1016/0021-9797(87)90360-2

[70] Kaler EW, Bennet KE, Davis HT and Scriven LE, Toward understanding microemulsion microstucture: a small-angle $\mathrm{x}$ ray scattering study. J Chem Phys 1983; 79: 5673-84. http://dx.doi.org/10.1063/1.445688 
[71] Eicke HF and Rehak J. On the formation of water/oil microemulsions. Helv Chim Acta 1976; 59: 2883-91. http://dx.doi.org/10.1002/hlca.19760590825

[72] Degiorgio V. Physics of amphiphiles: micelles, vesicles and microemulsions. North Holland Phys Pub; 1985.

[73] Pouchelon A, Chatenay D, Meunier J and Langevin D. Origin of low interfacial tensions in systems involving microemulsion phase. J Coll Int Sci 1981; 82: 418-22.

http://dx.doi.org/10.1016/0021-9797(81)90383-0

[74] Atik SS, and Thomas JK. Transport of photoproduced ions in water in oil microemulsions: Movement of ions from one water pool to another. J Amer Chem Soc 1981; 103: 354350 . http://dx.doi.org/10.1021/ja00402a048

[75] Fletcher PDI, Howe AM and Robinson BH. The kinetics of solubilisate exchange between water droplets of a water-inoil microemulsion. J Chem Soc Faraday Trans 1 1987; 83: 985-1006. http://dx.doi.org/10.1039/f19878300985

[76] Lang J, Jada A and Malliaris A. Structure and dynamics of water-in-oil droplets stabilized by sodium bis(2ethylhexyl)sulfosuccinate. J Phys Chem 1988; 92: 1946-53. http://dx.doi.org/10.1021/j100318a047

[77] Cabos C, Delord P. Study, by neutron small angle scattering, of addition of an electrolyte to reversed micellar solution. J Phys Lett 1980; 41: 455-8. http://dx.doi.org/10.1051/jphyslet:019800041018045500

[78] Kirkpatrick S. Classical transport in disordered media: Scaling and effective-medium theories. Phys Rev Lett 1971; 27: $1722-5$. http://dx.doi.org/10.1103/PhysRevLett.27.1722

[79] Kirkpatrick S. Percolation and Conduction. Rev Mod Phys 1973; 54: 574-88. http://dx.doi.org/10.1103/RevModPhys.45.574

[80] Webman J, Jortner $\mathrm{J}$ and Cohen MH. Numerical simulation of electrical conductivity in microscopically inhomogenous materials. Phys Rev B 1975; 11: 2885-92.

http://dx.doi.org/10.1103/PhysRevB.11.2885

[81] Granqvist CG and Hunderi O. Conductivity of homogeneous materials: Effective-medium theory with dipole-dipole interaction. Phys Rev B 1978; 18: 1554-61. http://dx.doi.org/10.1103/PhysRevB.18.1554

[82] Bernasconi $\mathrm{J}$ and Weisman HJ. Effective-medium theories for site-disordered resistance networks. Phys Rev B 1976; 13: 1131-9.

http://dx.doi.org/10.1103/PhysRevB.13.1131

[83] Paul S, Bisal S and Moulik SP. Physicochemical studies on microemulsions: test of the theories of percolation. J Phys Chem 1992; 96: 896-901.

http://dx.doi.org/10.1021/j100181a067

[84] Eicke HF, Borkovec M and Das-Gupta B. Conductivity of water-in-oil microemulsions: a quantitative charge fluctuation model. J Phys Chem 1989; 93: 314-7. http://dx.doi.org/10.1021/j100338a062

[85] Kallay $\mathrm{N}$ and Chittofrati A. Conductivity of microemulsions: refinement of charge fluctuation model. J Phys Chem 1990; 94: 4755-6.

http://dx.doi.org/10.1021/j100374a070

[86] Bhattacharya S, Stokes JP, Kim MW and Huang JS. Percolation in an oil-continuous microemulsion. Phys Rev Lett 1985; 55: 1884-7.

http://dx.doi.org/10.1103/PhysRevLett.55.1884

[87] Kim MW and Huang JS. Percolationlike phenomena in oilcontinuous microemulsions. Phys Rev A 1986; 34: 719-22. http://dx.doi.org/10.1103/PhysRevA.34.719

[88] Álvarez E, García-Río L, Mejuto JC and Navaza JM. Effects of temperature on the conductivity of sodium bis(2ethylhexyl)sulfosuccinate + 2,2,4-trimethylpentane + water microemulsions. Influence of amides and ethylene glycol. J Chem Eng Data 1999; 44: 484-7.

http://dx.doi.org/10.1021/je980129c

[89] Álvarez E, García-Río L, Mejuto JC, Navaza JM and PérezJuste J. Effects of temperature on the conductivity of microemulsions: Influence of sodium hydroxide and hydrochloric acid. J Chem Eng Data 1999; 44: 846-9. http://dx.doi.org/10.1021/je990026+

[90] Dasilva-Carvalhal J, García-Río L, Gómez-Díaz D, Mejuto JC and Navaza JM. Effect of temperature upon electrical conductivity of sodium bis(2-ethylhexyl) sulfosuccinate + 2,2,4-trimethylpentane + water + phase transfer catalyst. J Chem Eng Data 2006; 51: 1749-54. http://dx.doi.org/10.1021/je060162w

[91] Moha-Ouchane M, Peyrelasse J and Boned C. Percolation transition in microemulsions: Effect of water-surfactant ratio, temperature, and salinity. Phys Rev A 1987; 35: 3027-32. http://dx.doi.org/10.1103/PhysRevA.35.3027

[92] Mathew C, Patanjali PK, Nabi A and Maitra A. On the concept of percolative conduction in Water-in-Oil microemulsions. Coll Surf 1988; 30: 253-63.

http://dx.doi.org/10.1016/0166-6622(88)80210-5

[93] Jada A, Lang $\mathrm{J}$ and Zana R. Relation between electrical percolation and rate constant for exchange of material between droplets in water in oil microemulsions. J Phys Chem 1989; 93: 10-12.

http://dx.doi.org/10.1021/j100338a004

[94] Jada A, Lang J, Zana R, Makhloufi R, Hirsch E and Candau SJ. Ternary water in oil microemulsions made of cationic surfactants, water, and aromatic solvents. 2. Droplet sizes and interactions and exchange of material between droplets. J Phys Chem 1990; 94: 387-95.

http://dx.doi.org/10.1021/j100364a066

[95] García-Río L, Gómez-Díaz D, Mejuto JC, Navaza JM, Rodríguez-Álvarez A and Pérez-Lorenzo M. Percolative phenomena of AOT-based microemulsions in presence of organic acids. Rec Dev Coll Int Res 2004; 2: 35-45.

[96] García-Río L, Gómez-Díaz D, Mejuto JC, Pérez-Juste J and Rodríguez-Dafonte P. Internal dynamics and properties of water/AOT/isooctane microemulsions: effect of additives. Rec Res Dev Phys Chem 2002; 6: 213-40.

[97] Dasilva-Carvalhal J, García-Río L, Gómez-Díaz D, Mejuto JC and Rodríguez-Dafonte $P$. Influence of crown ethers on the electric percolation of AOT/Isooctane/Water (w/o) microemulsions. Langmuir 2003; 19: 5975-83. http://dx.doi.org/10.1021/la026857m

[98] García-Río L, Hervés P, Leis JR and Mejuto JC. Influence of crown ethers and macrocyclic cryptands upon the percolation phenomena in $\mathrm{AOT} /$ isooctane $/ \mathrm{H}_{2} \mathrm{O}$ microemulsions. Langmuir 1997; 13: 6083-8. http://dx.doi.org/10.1021/la970297n

[99] Hait SK, Sanyal A and Moulik SP. Physicochemical studies on microemulsions. 8. The effects of aromatic methoxy hydrotropes on droplet clustering and understanding of the dynamics of conductance percolation in Water/Oil microemulsion systems. J Phys Chem B 2002; 106: 1264250 .

http://dx.doi.org/10.1021/jp026702n

[100] Roy BK and Moulik SP. Functions of hydrotropes (sodium salicylate, proline, pyrogallol, resorcinol and urea) in solution with special reference to amphiphile behaviors. Coll Surf $A$ 2002; 203: 155-66. http://dx.doi.org/10.1016/S0927-7757(01)01099-8

[101] Hait SK, Moulik SP, Rodgers MP, Burke SE and Palepu R. Physicochemical studies on microemulsions. 7. Dynamics of percolation and energetics of clustering in Water/AOT/Isooctane and Water/AOT/Decane w/o microemulsions in presence of hydrotopes (sodium salicylate, $\alpha$-naphthol, $\beta$-naphthol, resorcinol, catechol, 
hydroquinone, pyrogallol and urea) and bile salt (sodium cholate). J Phys Chem B 2001; 105: 7145-54.

http://dx.doi.org/10.1021/jp0105084

[102] Moulik SP, De GC, Bhowmik BB and Panda AK. Physicochemical studies on microemulsions. 6. Phase behavior, dynamics of percolation, and energetics of droplet clustering in Water/AOT/n-Heptane system influenced by additives (sodium cholate and sodium salicylate). J Phys Chem B 1999; 103: 7122-9.

http://dx.doi.org/10.1021/jp990360c

[103] Ray S, Paul S and Moulik SP. Physicochemical studies on microemulsions $\mathrm{V}$. Additive effects on the performance of scaling equations and activation energy for percolation of conductance of Water/AOT/Heptane microemulsion. J Coll Int Sci 1996; 183: 6-12.

http://dx.doi.org/10.1006/jcis.1996.0512

[104] Ray S, Paul S and Moulik SP. Structure and dynamics of microemulsions. Part 1. Effect of additives on percolation of conductance and energetics of clustering in water-AOTheptane microemulsions. J Chem Soc Faraday Trans 1993; 89: $3277-82$

http://dx.doi.org/10.1039/ft9938903277

[105] García-Río L, Leis JR, Mejuto JC, Peña ME. and Iglesias E. Effects of additives on the internal dynamics and properties of Water/AOT/Isooctane microemulsions. Langmuir 1994; 10: 1676-83.

http://dx.doi.org/10.1021/la00018a013

[106] Finer EG, Franks $F$ and Tait MJ. Nuclear magnetic resonance studies of aqueous urea solutions. J Am Chem Soc 1972; 94: 4424-9. http://dx.doi.org/10.1021/ja00768a004

[107] Evans DF, Mitchell DJ and Ninham BW. Oil, water, and surfactant: properties and conjectured structure of simple microemulsions. J Phys Chem 1986; 90: 2817-25. http://dx.doi.org/10.1021/j100404a009

[108] Mejuto JC. Organic Reactivity in Microheterogeneous media. Doctoral Thesis. University of Santiago de Compostela; 1996.

[109] Chakraborty I and Moulik SP. Physicochemical studies on microemulsions 9. Conductance percolation of AOT-derived W/O microemulsion with aliphatic and aromatic hydrocarbon oils. J Coll Int Sci 2005; 289: 530-41.

http://dx.doi.org/10.1016/j.jcis.2005.03.080

[110] Mitra D, Chakraborty I, Bhattacharya SC, Moulik SP, Roy S, Das D and Das PK. Physicochemical studies on cetylammonium bromide and its modified (mono-, di-, and trihydroxyethylated) head group analogues. Their micellization characteristics in water and thermodynamic and structural aspects of water-in-oil microemulsions formed with them along with $\mathrm{n}$-hexanol and isooctane. J Phys Chem B 2006; 110: 11314-26.

http://dx.doi.org/10.1021/jp055720c

[111] Álvarez E, García-Río L, Mejuto JC and Navaza JM. Effects of temperature on the conductivity of sodium bis(2ethylhexyl) sulfosuccinate + 2,2,4-trimethylpentane + water microemulsions. Influence of sodium salts. J Chem Eng Data 1998; 43:519-22.

http://dx.doi.org/10.1021/je970238b

[112] Álvarez E, García-Río L, Mejuto JC, Navaza JM and PérezJuste J. Effects of temperature on the conductivity of AOT/isooctane/water microemulsions. Influence of salts. J Chem Eng Data 1999; 44: 850-3.

http://dx.doi.org/10.1021/je9900575

[113] Bravo C, Hervés P, Leis JR and Peña ME. Micellar effects in the acid denitrosation of N-nitroso-N-methyl-ptoluenesulfonamide. J Phys Chem 1990; 94: 8816-20. http://dx.doi.org/10.1021/j100388a014

[114] He Z, O'Connor PJ, Romsted LS and Zanette DJ. Specific counterion effects on indicator equilibria in micellar solutions of decyl phosphate and lauryl sulfate surfactants. J Phys Chem 1989; 93: 4219-26.

http://dx.doi.org/10.1021/j100347a064

[115] Lemaire B, Bothorel P and Roux D. Micellar interactions in water-in-oil microemulsions. 1. Calculated interaction potential. J Phys Chem 1983; 87: 1023-8. http://dx.doi.org/10.1021/j100229a021

[116] Brunetti S, Roux D, Mellocq AM, Fourche G and Bothorel P. Micellar interactions in water-in-oil microemulsions. 2. Light scattering determination of the second virial coefficient. J Phys Chem 1983; 87: 1028-34. http://dx.doi.org/10.1021/j100229a022

[117] Hou M, Shah DO. Effects of the molecular structure of the interface and continuous phase on solubilization of water in water/oil microemulsions, Langmuir 1987; 3 1086-96. http://dx.doi.org/10.1021/la00078a036

[118] Robson-Wright M. The Nature of Electrolyte Solutions. MacMillan Education; 1988.

[119] García-Rio L. Studies on the reactivity of nitrosocompounds in water, organics solvents and microheterogeneous media. Doctoral Thesis. University of Santiago de Compostela; 1993.

[120] Álvarez E, García-Río L, Leis JR, Mejuto JC and Navaza JM. Effect of the temperature on the conductivity of sodium bis(2ethylhexyl)sulfosuccinate $+2,2,4$-trimethylpentane + water microemulsions in the presence of ureas and thioureas. $J$ Chem Eng Data 1998; 43: 123-7. http://dx.doi.org/10.1021/je970104y

[121] Fang $\mathrm{J}$ and Venable RL. Conductivity study of the microemulsion system sodium dodecyl sulfate-hexylamineheptane-water. J Coll Int Sci 1987; 116: 269-77. http://dx.doi.org/10.1016/0021-9797(87)90120-2

[122] Swenson CA. Effects of protein denaturants of the ureaguanidinium class on bulk water structure: An infrared study. Arch Biochem Biophys 1966; 117: 494-8. http://dx.doi.org/10.1016/0003-9861(66)90088-9

[123] Sasaki K and Arakawa K. The ultrasonic study of aqueous solutions of alkyl-substituted urea. Bull Chem Soc Jpn 1969; 42: $2485-9$.

http://dx.doi.org/10.1246/bcsj.42.2485

[124] Kuharski RA and Rossky P. Molecular dynamics study of solvation in urea water solution. J Am Chem Soc 1984; 106 5786-93.

http://dx.doi.org/10.1021/ja00332a005

[125] Cristinziano P, Lelj F, Amoedo P, Barone G and Barone V. Stability and structure of formamide and urea dimers in aqueous solution. A theoretical study. J Chem Soc Faraday Trans 1 1989; 85: 621-32.

http://dx.doi.org/10.1039/f19898500621

[126] Subramanian D, Sarma TS, Balasubramanian D and Ahluwalia JC. Effects of the urea-guanidinium class of protein denaturation on water structure: heats of solution and proton chemical shift studies. J Phys Chem 1971; 75: 815-20. http://dx.doi.org/10.1021/j100676a016

[127] Costa-Amaral CL, Brino O, Chaimovich $\mathrm{H}$ and Politi JM Formation and properties of reversed micelles of Aerosol OT containing urea in the aqueous pool. Langmuir 1992; 8: 2417-21.

http://dx.doi.org/10.1021/la00046a013

[128] Kang YS, McManus HJD and Kevan L. An electron magnetic resonance study on the photoionization of $\mathrm{N}$ alkylphenothiazines in dioctadecyldimethylammonium chloride frozen vesicles: the effect of urea, 1,3-dimethylurea, 1,3-diethylurea, and 1,1',3,3'-tetramethylurea. Phys Chem 1992; 96: 10055-60.

http://dx.doi.org/10.1021/j100203a085

[129] Moulik SP, Digout LG, Aylward WM and Palepu R. Studies on the interfacial composition and thermodynamic properties of W/O microemulsions. Langmuir 2000; 16: 3101-6. http://dx.doi.org/10.1021/la991028v 
[130] Álvarez E, García-Río L, Leis JR, Mejuto JC and Navaza JM. Effect of temperature on the conductivity of sodium bis(2ethylhexyl) sulfosuccinate + 2,2,4-trimethylpentane + water microemulsions. Influence of Amines. J Chem Eng Data 1998; 43: 433-5. http://dx.doi.org/10.1021/je970232m

[131] Giammona G, Goffredi F, Liveri F, Turco V and Vassallo G. Water structure in water/AOT/n-heptane microemulsions by FTIR spectroscopy. J Coll Int Sci 1992; 154: 411-5. http://dx.doi.org/10.1016/0021-9797(92)90156-G

[132] García-Río L, Hervés P, Mejuto JC, Pérez-Juste $J$ and Rodríguez-Dafonte P. Effects of alkylamines on the percolation phenomena in water/AOT/isooctane microemulsions. J Coll Int Sci 2000; 225: 259-64. http://dx.doi.org/10.1006/jcis.2000.6771

[133] Álvarez E, García-Río L, Leis JR "et al.". Effect of temperature on the electrical conductivity of sodium bis(2ethylhexyl)sulfosuccinate + 2,2,4-trimethylpentane + water microemulsions. Influence of alkylamines. J Chem Eng Data 1999; 44: 1286-90.

http://dx.doi.org/10.1021/je990108y

[134] García-Río L, Hervés P, Mejuto JC, Pérez-Juste J and Rodríguez-Dafonte P. Pseudophase approach to reactivity in microemulsions: Quantitative explanation of the kinetics of the nitroso group transfer reactions between $\mathrm{N}$-methyl- $\mathrm{N}$ nitroso-p- toluenesulfonamide and secondary alkylamines in Water/AOT/Isooctane microemulsions. Ind Eng Chem Res 2003; 44: 5450-6.

http://dx.doi.org/10.1021/ie0208523

[135] García-Río L, Mejuto JC, Pérez-Lorenzo M, RodríguezÁlvarez $\mathrm{A}$ and Rodríguez-Dafonte $\mathrm{P}$. Influence of anionic surfactants on the electric percolation of AOT/isooctane/water microemulsions. Langmuir. 2005; 21: 6259-64. http://dx.doi.org/10.1021/la0501987

[136] Eicke HF and Meier W. Nonmonotonic pattern of the critical percolation temperature due to variations of additive chain length in water-in-oil microemulsions. Coll Polym Sci 2001; 279: $301-4$

http://dx.doi.org/10.1007/s003960000462

[137] Eicke HF. Nonmonotonic electric conductivity by alkyl-chain variation of an ionic additive in percolated nonionic W/Omicroemulsions. J Phys Chem B. 2001; 105: 2753-6. http://dx.doi.org/10.1021/jp0041003

[138] Cid-Samamed A, García-Río L, Fernández-Gándara D, Mejuto JC, Morales $\mathrm{J}$ and Pérez-Lorenzo M. Influence of $\mathrm{n}$ alkyl acids on the percolative phenomena in AOT-based microemulsions. J Coll Int Sci 2008; 318: 525-9. http://dx.doi.org/10.1016/j.jcis.2007.11.001
[139] Nazário LMM, Crespo JPSG, Holzwarth JF and Hatton TA. Dynamics of AOT and AOT/nonionic cosurfactant microemulsions. An lodine-Laser temperature jump study. Langmuir 2000; 16: 5892-9.

http://dx.doi.org/10.1021/la991674u

[140] Menger FM. Structure of micelles. Acc Chem Res 1979; 12 : 111-7. http://dx.doi.org/10.1021/ar50136a001

[141] Bravo C, García-Río L, Leis JR, Peña ME and Iglesias EJ. A ${ }^{1} \mathrm{H}$ NMR study of the location of nitroso compounds and penetration of water in micellar aggregates. Coll Int Sci 1994; 166: 316-20.

http://dx.doi.org/10.1006/jcis.1994.1301

[142] Ruasse M-F, Blagoeva IB, Ciri R, et al. Organic reactions in micro-organized media: Why and how? Pure Appl Chem 1997; 69: 1923-32.

http://dx.doi.org/10.1351/pac199769091923

[143] García-Río L, Herves P, Mejuto JC, Perez-Juste J and Rodríguez-Dafonte P. Pseudophase approach to reactivity in microemulsions: Quantitative explanation of the kinetics of the nitroso group transfer reactions between $\mathrm{N}$-methyl- $\mathrm{N}$ nitroso-p- toluenesulfonamide and secondary alkylamines in Water/AOT/Isooctane microemulsions. Ind Eng Chem Res 2003; 42: 5450-6. http://dx.doi.org/10.1021/ie0208523

[144] García-Río L, Hervés P, Mejuto JC and Rodríguez-Dafonte $P$. Nitrosation reactions in Water/AOT/Xylene microemulsions. Ind Eng Chem Res 2006; 45: 600-6. http://dx.doi.org/10.1021/ie050925t

[145] García-Río L, Leis JR, Mejuto JC and Pérez-Lorenzo M. Microemulsions as microreactors in physical organic chemistry. Pure Appl Chem 2007; 79: 1111-23. http://dx.doi.org/10.1351/pac200779061111.

[146] Astray G, Cid A, García-Río L, Hervella P, Mejuto JC and Pérez-Lorenzo M. Organic reactivity in AOT-stabilized microemulsions. Prog React Kinet Mech 2008; 33: 81-97. http://dx.doi.org/10.3184/146867807X273173

[147] Stamatis H, Xenakis A, Menge $U$ and Kolisis FN. Kinetic study of lipase catalyzed esterification reactions in water-iniol microemulsions. Biotechnol Bioeng 1993; 42: 931-7. http://dx.doi.org/10.1002/bit.260420803

[148] Stamatis H, Xenakis A, Dimitriadis E and Kolisis FN Catalytic behavior of Pseudomonas cepacia lipase in w/o microemulsions. Biotechnol Bioeng 1995; 45: 33-41. http://dx.doi.org/10.1002/bit.260450106

[149] Kolisis FN, Valis TP and Xenakis A. Lipase-catalyzed esterification of fatty acids in nonionic microemulsions. Ann New York Acad Sci 1990; 613: 674-80. http://dx.doi.org/10.1111/j.1749-6632.1990.tb18244.x

\section{DOI: http://dx.doi.org/10.6000/1929-5030.2014.03.02.8}

(C) 2014 Cid et al.; Licensee Lifescience Global.

This is an open access article licensed under the terms of the Creative Commons Attribution Non-Commercial License (http://creativecommons.org/licenses/by-nc/3.0/) which permits unrestricted, non-commercial use, distribution and reproduction in any medium, provided the work is properly cited. 